RESCEU-28/11

ICRR-Report-592-2011-9

IPMU11-0122

TU-885

\title{
Non-Gaussianity from Curvatons Revisited
}

\author{
Masahiro Kawasaki* ${ }^{* 1}$, Takeshi Kobayashi ${ }^{\ddagger 2}$, and Fuminobu Takahashi ${ }^{\dagger, * 3}$ \\ * Institute for Cosmic Ray Research, The University of Tokyo, \\ 5-1-5 Kashiwanoha, Kashiwa, Chiba 277-8582, Japan \\ $\dagger$ Institute for the Physics and Mathematics of the Universe, The University of Tokyo, \\ 5-1-5 Kashiwanoha, Kashiwa, Chiba 277-8582, Japan \\ $\ddagger$ Research Center for the Early Universe, School of Science, The University of Tokyo, \\ 7-3-1 Hongo, Bunkyo-ku, Tokyo 113-0033, Japan \\ * Department of Physics, Tohoku University, Sendai 980-8578, Japan
}

\begin{abstract}
We investigate density perturbations sourced by a curvaton with a generic energy potential. The key feature of a curvaton potential which deviates from a quadratic is that the curvaton experiences a non-uniform onset of its oscillation. This sources additional contributions to the resulting density perturbations, and we especially find that the non-Gaussianity parameter $f_{\mathrm{NL}}$ can become large with either sign no matter whether the curvaton dominates or subdominates the universe when it decays. Such non-quadratic curvaton potentials are required in order to produce a red-tilted density perturbation spectrum (without invoking large-field inflation), and are also motivated by explicit curvaton models based on microscopic physics. We further apply our generic results to the case where the curvaton is a pseudo-Nambu-Goldstone (NG) boson of a broken $\mathrm{U}(1)$ symmetry, and show that the resulting density perturbations are strongly enhanced towards the hilltop region of the energy potential, accompanied by a mild increase of the non-Gaussianity. Such hilltop NG curvatons can produce observationally suggested density perturbations under wide ranges of inflation/reheating scales, and further predict the non-Gaussianity of the density perturbations to lie within the range $10 \lesssim f_{\mathrm{NL}} \lesssim 30$.
\end{abstract}

\footnotetext{
${ }^{1}$ kawasaki@icrr.u-tokyo.ac.jp

${ }^{2}$ takeshi-kobayashi@resceu.s.u-tokyo.ac.jp

${ }^{3}$ fumi@tuhep.phys.tohoku.ac.jp
} 


\section{Contents}

1 Introduction $\quad 1$

2 Density Perturbations from Curvatons 4

2.1 Computing $\delta \mathcal{N} \ldots \ldots \ldots \ldots \ldots \ldots$

2.1.1 Case $t_{\mathrm{reh}}<t_{\mathrm{osc}} \ldots \ldots \ldots \ldots \ldots$. . . . . . . . . . . . . . . . . . . . . . . . . . .

2.1.2 Case $t_{\mathrm{reh}}>t_{\mathrm{osc}} \ldots \ldots \ldots \ldots . \ldots \ldots$

2.2 Curvaton Field Value at the Onset of Oscillation . . . . . . . . . . . . . 8

2.3 When the Curvaton Starts Oscillating . . . . . . . . . . . . . . . 9

2.4 Results . . . . . . . . . . . . . . . . . . . . . . . 10

2.5 Validity of the Analytic Expressions . . . . . . . . . . . . . . . . . . 12

2.6 Spectral Index . . . . . . . . . . . . . . . . . . . . . . 13

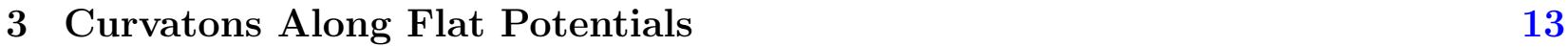

3.1 Hilltop Curvatons . . . . . . . . . . . . . . . . . . . . . . . 14

4 Case Study: Pseudo-Nambu-Goldstone Curvatons 16

4.1 Density Perturbations from NG Curvatons . . . . . . . . . . . . . . . 17

4.1 .1 Non-Hilltop Region . . . . . . . . . . . . . . . . . . . . . 17

4.1 .2 Hilltop Region . . . . . . . . . . . . . . . . . . . . . . . 19

4.2 Parameter Space . . . . . . . . . . . . . . . . . . . . . . . 20

4.2.1 Requirements . . . . . . . . . . . . . . . . . 22

4.2.2 Windows for Inflation/Reheating Scales . . . . . . . . . . . . . . . . 23

4.3 Discussion on Hilltop Curvatons . . . . . . . . . . . . . . . . . . . . . . . 28

5 Conclusions $r$

A Scalar Field Dynamics in an Expanding Universe 30

B Density Perturbations from Curvatons with Non-Sinusoidal Oscillations 31

\section{Introduction}

The origin of the large-scale structures of our universe can be explained by the presence of primordial density perturbations in the early universe, which were of super-horizon scales and almost scale-invariant. However, understanding how such density perturbations were seeded has remained a big mystery for cosmology. On the other hand, cosmic inflation $[1,2,3]$ has been known to be able to generate super-horizon and (nearly) scale-invariant fluctuations for scalar fields. (Early universe models that can generate such field fluctuations without having an inflationary era have also been studied, see e.g. $[4,5,6]$.) Then now, one may rephrase 
the question about the origin of the large-scale structures as how such field fluctuations were converted into the primordial density perturbations of the universe, which eventually lead to structure formation.

An attractive mechanism for creating the primordial density perturbations from the field fluctuations is the curvaton mechanism [7, 8, 9, 10], in which the curvaton field possessing large-scale field fluctuations generates the density perturbations while it oscillates about its potential minimum and comes close to dominating the universe. The idea of the curvaton mechanism is thus simple, and also has the merit that when embedded in inflationary cosmology, it frees the inflaton from being responsible for generating the perturbations and therefore drastically relaxes constraints on inflationary model building. However, while there have been extensive works on the curvaton mechanism, most of the literature has focused on curvatons possessing rather trivial potentials (e.g. quadratic potentials), thus lead to common predictions for the density perturbations, such as large/small non-Gaussianity in the perturbations depending on whether the curvaton is subdominant/dominant upon decay.

In this paper, we explore density perturbations sourced by a curvaton rolling along an arbitrary energy potential. The key feature of a curvaton potential which deviates from a quadratic one is that the curvaton field with large-scale field fluctuations starts its oscillation at different times at different patches of the universe. Such behaviour contributes to the curvaton energy density perturbations in addition to that sourced directly from the original field fluctuations. We find that such non-trivial conversion processes of the field fluctuations into the density perturbations can lead to strong enhancement/suppression of the density perturbations of the universe, as well as for their non-Gaussianities. In particular, we will show that the non-Gaussianity parameter $f_{\mathrm{NL}}$ can become large with either sign no matter if the curvaton dominates or subdominates the universe when it decays. Several previous works have considered specific cases, e.g., curvaton potentials with a non-quadratic polynomial term were studied in $[11,12,13]$, and cosine type potentials in [14] which clearly pointed out that non-uniform onset of curvaton oscillations can have important effects on the density perturbations. Related discussions can be found in [15], though such effects were not explicitly taken into account. In this paper we carry out a systematic study of density perturbations from curvatons with generic potentials through analytic analyses.

It is worthwhile to investigate curvaton potentials which deviate from simple quadratic ones, both from observational and theoretical reasons: Considering a curvaton whose field fluctuations were generated during the inflationary era (which is the case studied in this paper), in order for the curvaton to produce the density perturbation spectrum which is red-tilted (i.e. with negative spectral index $n_{s}-1$ ) as suggested by latest CMB observations $[16,17,18]$ without relying on specific inflation mechanisms, ${ }^{4}$ the curvaton needs to be located along a potential with negative curvature during inflation (cf. (2.45)). This obviously suggests the cur-

\footnotetext{
${ }^{4}$ For a non-tachyonic curvaton (i.e. a curvaton located along a potential with non-negative curvature during inflation), the spectral index of the resulting density perturbation spectrum is set by the time variation of the Hubble parameter during inflation as $n_{s}-1 \geq 2 \dot{H} / H^{2}$ (cf. (2.45)). Hence, assuming single-field canonical
} 
vaton potential to take non-trivial forms. Furthermore, explicit curvaton models constructed in the framework of microscopic physics can naturally possess intricate energy potentials, e.g., curvatons in supersymmetric models [20], a stringy curvaton model in [21] from a D-brane moving along the internal compactified space giving rise to a multi-dimensional periodic curvaton potential. [22] also discusses a string realization of the curvaton, where the potential is a linear combination of exponential terms.

We also apply our general results to the case where the curvaton is a pseudo-NambuGoldstone (NG) boson [23] of a broken U(1) symmetry. The periodic curvaton potential necessarily possesses not only minima but also maxima, around which the potential curvature is negative so that the resulting density perturbation spectrum can become red-tilted. We explore the parameter space of NG curvatons producing a red-tilted density perturbation spectrum as suggested by observations, and show that the allowed regions for the inflationary energy scale and the reheating temperature (i.e. when the inflaton decays) are strongly constrained, unless the NG curvaton is located close to the maximum of its potential during inflation. In such hilltop cases, the resulting density perturbations are extremely enhanced, thus relaxing the bounds on the inflation/reheating scales. Non-Gaussianity of the density perturbations also increases in the hilltop limit to values $10 \lesssim f_{\mathrm{NL}} \lesssim 30$ in most part of the allowed parameter window, which will be tested by upcoming CMB measurements. Such mild increase of non-Gaussianity towards the hilltop limit is actually a rather generic feature of hilltop curvatons, as will be shown in the paper.

This paper is organized as follows. We derive analytic expressions for density perturbations from curvatons with generic potentials in Section 2. Then in Section 3, we move on to discuss special cases where the curvaton starts its oscillation from a flat region of its potential, including hilltop curvatons. It will be shown that in such cases, effects due to fluctuations of the onset of the oscillation become crucial. As a simple example of a curvaton model generating density perturbations that match with observational constraints, we investigate the case where the curvaton is a pseudo-NG boson in Section 4. We present our conclusions in Section 5. Discussions on dynamics of scalar fields in an expanding universe are provided in Appendix A. While we focus on sinusoidal curvaton oscillations in the main body of the paper, in Appendix B we further generalize our discussions to incorporate non-sinusoidal curvaton oscillations. Curvatons along linear potentials are also discussed in Appendix B.

slow-roll inflation, the Lyth bound [19] relates the spectral index with the inflaton field $\phi$ range as

$$
\frac{1}{M_{p}}\left|\frac{d \phi}{d \mathcal{N}}\right| \geq \sqrt{1-n_{s}}
$$

where $M_{p}$ is the reduced Planck mass and $\mathcal{N}$ the e-folding number. This shows that unless the curvaton is tachyonic during inflation, the WMAP [16] central value $n_{s} \approx 0.96$ requires a super-Planckian field range for the inflaton. 


\section{Density Perturbations from Curvatons}

In this paper we focus on curvatons $[7,8,9,10,24]$ whose field fluctuations are generated in the inflationary era (though many features and results can be shared with curvatons with noninflationary fluctuation generating scenarios). Then a curvaton should be a light field so that it acquires field fluctuations that are nearly scale-invariant and Gaussian. Its energy density is considered to be initially negligible, and after inflation the curvaton starts oscillating about its potential minimum and behaves as nonrelativistic matter. As its energy density grows relative to radiation sourced from the inflaton decay, the curvaton fluctuations increasingly contribute to the density perturbations until the curvaton decays or dominates the universe.

One of the main points of this paper is that the original field fluctuations of the curvaton $\sigma$ during inflation can give rise to perturbations of the curvaton energy density through perturbing the time when the curvaton starts its oscillation, as well as by directly perturbing its energy density $\rho_{\sigma}$. Let us schematically write down the resulting density perturbations of the universe as

$$
\zeta \sim c_{1} \frac{\delta \rho_{\sigma}}{\rho_{\sigma}}-c_{2} \frac{\delta H_{\mathrm{osc}}}{H_{\mathrm{osc}}}
$$

where $H_{\text {osc }}$ is the Hubble parameter at the onset of the curvaton oscillation. We have added a minus sign to the second term in the right hand side to indicate that larger $H_{\text {osc }}$ gives an earlier onset of the curvaton oscillation, which more quickly redshifts away the curvaton energy density. (2.1) illustrates that direct energy perturbations $\delta \rho_{\sigma}$ can be cancelled by effects from $\delta H_{\text {osc }}$, suppressing the linear order density perturbations with possible enhancement of the non-Gaussianity. Moreover, when effects from $\delta H_{\text {osc }}$ become dominant over that from $\delta \rho_{\sigma}$, the resulting density perturbations become very different from cases with the familiar quadratic curvaton potential. However we should also stress that (2.1) is rather schematic, and the actual density perturbation spectrum is expressed as an involved combination of the two effects, cf. (2.39).

In the following subsections, we explicitly compute the density perturbations from curvatons with generic energy potentials. First deriving an expression of the form similar to (2.1) using the $\delta \mathcal{N}$-formalism $[25,26,27,28]$, we then analyze the curvaton dynamics and obtain analytic expressions for the density perturbations.

We suppose that the Hubble parameter $H=\dot{a} / a$ (with $a$ being the scale factor of the universe, and an overdot denoting a time derivative) is nearly constant during inflation, and that at the end of inflation, the inflaton $\phi$ suddenly turns into matter, i.e. its energy density starts decreasing as $\rho_{\phi} \propto a^{-3}$, and then at reheating, suddenly decays into radiation, i.e. starts decreasing as $\rho_{r} \propto a^{-4}$. Curvature perturbations sourced from the inflaton are ignored. Furthermore, upon carrying out analytic estimations, we make the following simplifying assumptions for the curvaton:

1. The curvaton $\sigma$ starts a sinusoidal oscillation at some time after inflation, from then 


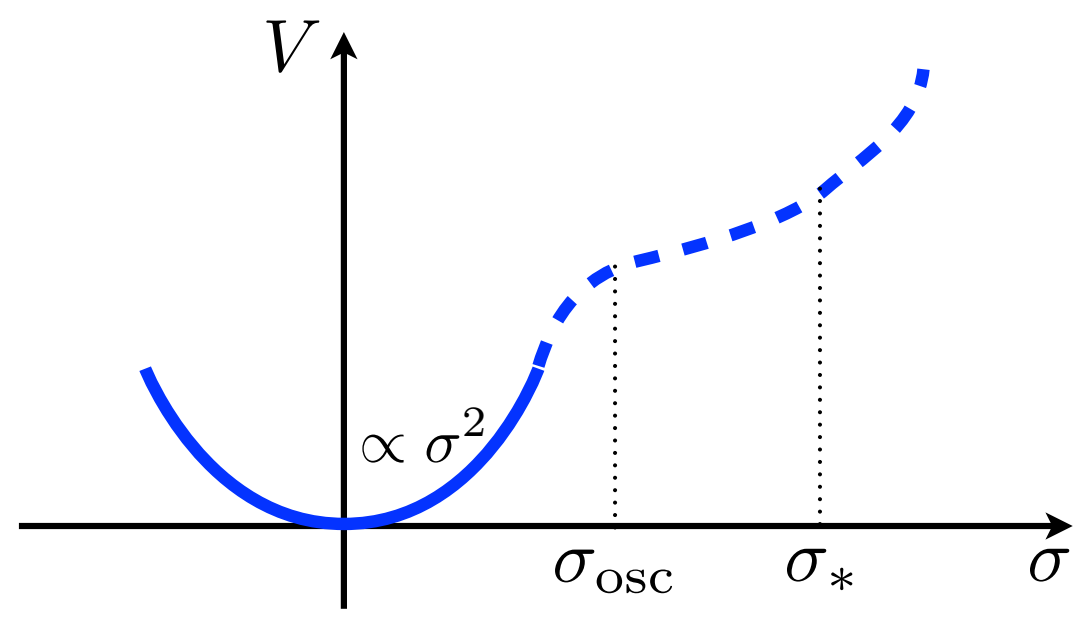

Figure 1: Schematic of the curvaton potential under consideration: The curvaton rolls along its energy potential following an attractor solution (2.24), then it starts oscillating about the (local) minimum. The potential can take arbitrary forms, but once the curvaton starts the oscillation (from $\sigma_{\text {osc }}$ ) its amplitude soon decays and the potential is well approximated by a quadratic one.

we treat the curvaton as matter. (Hence we are considering cases where the curvaton potential is well approximated by quadratic close to its (local) minimum, and that the curvaton oscillation happens mostly in the quadratic regime, cf. Figure 1. However, one can straightforwardly generalize the calculations to other cases, as is discussed in Appendix B.)

2. The energy density of the curvaton until it starts oscillating is so tiny compared to the total energy density that its effect on the expansion of the universe is negligible at least until the onset of the curvaton oscillation.

The curvaton acting as matter is also assumed to suddenly decay into radiation when $H=\Gamma_{\sigma}$, where we take the curvaton decay rate $\Gamma_{\sigma}$ to be a uniform constant independent of the curvaton field value.

\subsection{Computing $\delta \mathcal{N}$}

The curvature perturbations $\zeta$ from a curvaton can be computed using the $\delta \mathcal{N}$-formalism $[25$, $26,27,28]$, as the difference in the e-folding number $\mathcal{N}$ at a constant energy hypersurface, among different patches of the universe which took different field values of the curvaton at the time when the CMB scale exited the horizon during inflation, i.e.,

$$
\zeta(\boldsymbol{x})=\frac{\partial \mathcal{N}}{\partial \sigma_{*}} \delta \sigma_{*}(\boldsymbol{x})+\frac{1}{2} \frac{\partial^{2} \mathcal{N}}{\partial \sigma_{*}^{2}}\left(\delta \sigma_{*}(\boldsymbol{x})^{2}-\left\langle\delta \sigma_{*}(\boldsymbol{x})^{2}\right\rangle\right)+\cdots
$$


Here, we are assuming a homogeneous and isotropic background, and values at the horizon exit are denoted by the subscript $*$. Expanding $\zeta(\boldsymbol{x})$ as

$$
\zeta_{\boldsymbol{k}}=\int d^{3} \boldsymbol{x} e^{-i \boldsymbol{k} \cdot \boldsymbol{x}} \zeta(\boldsymbol{x})
$$

their two- and three-point correlation functions take the form

$$
\begin{gathered}
\left\langle\zeta_{\boldsymbol{k}_{\mathbf{1}}} \zeta_{\boldsymbol{k}_{\mathbf{2}}}\right\rangle=(2 \pi)^{3} \delta^{(3)}\left(\boldsymbol{k}_{\mathbf{1}}+\boldsymbol{k}_{\mathbf{2}}\right) P_{\zeta}\left(k_{1}\right), \\
\left\langle\zeta_{\boldsymbol{k}_{\mathbf{1}}} \zeta_{\boldsymbol{k}_{\mathbf{2}}} \zeta_{\boldsymbol{k}_{\mathbf{3}}}\right\rangle=(2 \pi)^{3} \delta^{(3)}\left(\boldsymbol{k}_{\mathbf{1}}+\boldsymbol{k}_{\mathbf{2}}+\boldsymbol{k}_{\mathbf{3}}\right) B_{\zeta}\left(k_{1}, k_{2}, k_{3}\right),
\end{gathered}
$$

where $k_{i} \equiv\left|\boldsymbol{k}_{\boldsymbol{i}}\right|$. We define the power spectrum of the density perturbations $\mathcal{P}_{\zeta}$ as

$$
P_{\zeta}(k)=\frac{2 \pi^{2}}{k^{3}} \mathcal{P}_{\zeta}(k)
$$

and also the non-linearity parameter $f_{\mathrm{NL}}[29]$ as

$$
B_{\zeta}\left(k_{1}, k_{2}, k_{3}\right)=\frac{6}{5} f_{\mathrm{NL}}\left(k_{1}, k_{2}, k_{3}\right)\left[P_{\zeta}\left(k_{1}\right) P_{\zeta}\left(k_{2}\right)+P_{\zeta}\left(k_{2}\right) P_{\zeta}\left(k_{3}\right)+P_{\zeta}\left(k_{3}\right) P_{\zeta}\left(k_{1}\right)\right],
$$

which parametrizes the non-Gaussianity of the density perturbations encoded in the threepoint function. From (2.2), these values can be evaluated in terms of the derivatives of $\mathcal{N}$, at the leading order in $\delta \sigma_{* \boldsymbol{k}}$, as

$$
\begin{gathered}
\mathcal{P}_{\zeta}=\left(\frac{\partial \mathcal{N}}{\partial \sigma_{*}}\right)^{2} \mathcal{P}_{\delta \sigma_{*}}, \\
f_{\mathrm{NL}}=\frac{5}{6} \frac{\partial^{2} \mathcal{N}}{\partial \sigma_{*}^{2}}\left(\frac{\partial \mathcal{N}}{\partial \sigma_{*}}\right)^{-2},
\end{gathered}
$$

where $\mathcal{P}_{\delta \sigma_{*}}$ denotes the power spectrum of the curvaton field fluctuations defined similarly as in (2.6). Moreover, we have considered the intrinsic non-Gaussianity of the field fluctuations to be negligibly small and thus ignored the three-point function for $\delta \sigma_{*}$.

\subsubsection{Case $t_{\text {reh }}<t_{\text {osc }}$}

Let us begin by studying the case where reheating (= inflaton decay, at $t_{\text {reh }}$ ) happens before the onset of the curvaton oscillation at $t_{\mathrm{osc}}$. In other words, the universe is radiation dominated when the curvaton starts oscillating. (Hereafter, values at time $t_{i}(i=$ reh, osc, etc.) are denoted with the same subscript.) We would like to derive the $\sigma_{*}$-dependence of the e-folding number

$$
\mathcal{N}=\mathcal{N}_{a}+\mathcal{N}_{b}
$$

where

$$
\mathcal{N}_{a} \equiv \int_{t_{*}}^{t_{\mathrm{osc}}} H\left(t^{\prime}\right) d t^{\prime}, \quad \mathcal{N}_{b} \equiv \int_{t_{\mathrm{osc}}}^{t_{\mathrm{dec}}} H\left(t^{\prime}\right) d t^{\prime}
$$


Here $t_{\text {dec }}$ denotes the time when the curvaton decays, i.e. when $H$ becomes equal to $\Gamma_{\sigma}$ (which is independent of $\sigma_{*}$ ). After $t_{\mathrm{dec}}$, the universe is filled with a single component, i.e. radiation, hence no extra $\delta \mathcal{N}$ is produced.

The energy density of radiation redshifts away as $\dot{\rho}_{r}=-4 H \rho_{r}$, thus

$$
\mathcal{N}_{a}=\int_{t_{*}}^{t_{\mathrm{reh}}} H\left(t^{\prime}\right) d t^{\prime}+\int_{t_{\mathrm{reh}}}^{t_{\mathrm{osc}}} H\left(t^{\prime}\right) d t^{\prime}=\mathrm{const} .+\frac{1}{4} \ln \frac{\rho_{\text {rreh }}}{\rho_{\text {rosc }}},
$$

where we have especially used Assumption 2. Since $3 M_{p}^{2} H_{\mathrm{osc}}^{2}=\rho_{\text {rosc }}$ (where $M_{p}$ is the reduced Planck mass), we get

$$
\frac{\partial \mathcal{N}_{a}}{\partial \sigma_{*}}=-\frac{1}{4} \frac{\partial}{\partial \sigma_{*}} \ln H_{\mathrm{osc}}^{2}
$$

Similarly, one obtains

$$
\mathcal{N}_{b}=\frac{1}{4} \ln \frac{\rho_{\text {rosc }}}{\rho_{r \mathrm{dec}}}=\frac{1}{4} \ln \frac{3 M_{p}^{2} H_{\mathrm{osc}}^{2}}{3 M_{p}^{2} \Gamma_{\sigma}^{2}-\rho_{\sigma \mathrm{dec}}},
$$

and by further using

$$
\rho_{\sigma \mathrm{dec}}=\rho_{\sigma \mathrm{osc}} e^{-3 \mathcal{N}_{b}}
$$

one can check that

$$
\frac{\partial \mathcal{N}_{b}}{\partial \sigma_{*}}=\frac{1}{4+3 r}\left(r \frac{\partial}{\partial \sigma_{*}} \ln \rho_{\sigma \mathrm{osc}}+\frac{\partial}{\partial \sigma_{*}} \ln H_{\mathrm{osc}}^{2}\right) .
$$

Here we have introduced

$$
\left.r \equiv \frac{\rho_{\sigma}}{\rho_{r}}\right|_{\mathrm{dec}}
$$

Therefore, from (2.13) and (2.16) we arrive at

$$
\frac{\partial \mathcal{N}}{\partial \sigma_{*}}=\frac{r}{4+3 r} \frac{\partial}{\partial \sigma_{*}}\left(\ln \rho_{\sigma \mathrm{osc}}-\frac{3}{4} \ln H_{\mathrm{osc}}^{2}\right),
$$

which reproduces the schematic expression of (2.1). We note that the second term in the right hand side that is due to the non-uniform onset of the curvaton oscillations, can become important for non-quadratic curvaton potentials. ${ }^{5}$

Computation of the second derivative of $\mathcal{N}$ is also straightforward. Since

$$
r=\left.\frac{\rho_{\sigma}}{\rho_{r}}\right|_{\mathrm{osc}} e^{\mathcal{N}_{b}}
$$

one finds

$$
\frac{\partial r}{\partial \sigma_{*}}=4(1+r) \frac{\partial \mathcal{N}}{\partial \sigma_{*}}
$$

thus giving

$$
\frac{\partial^{2} \mathcal{N}}{\partial \sigma_{*}^{2}}=\frac{16(1+r)}{(4+3 r) r}\left(\frac{\partial \mathcal{N}}{\partial \sigma_{*}}\right)^{2}+\frac{r}{4+3 r} \frac{\partial^{2}}{\partial \sigma_{*}^{2}}\left(\ln \rho_{\sigma \text { osc }}-\frac{3}{4} \ln H_{\text {osc }}^{2}\right) .
$$

\footnotetext{
${ }^{5}$ Strictly speaking, the first term also contains effects due to non-uniform onset of the oscillations through $\partial \sigma_{\mathrm{osc}} / \partial \sigma_{*}$, as we will see later. Effects from $\delta H_{\mathrm{osc}}$ displayed in (2.18) have not been taken into account explicitly in previous works giving general discussions on density perturbations in the curvaton scenario.
} 


\subsubsection{Case $t_{\mathrm{reh}}>t_{\mathrm{osc}}$}

The case of $t_{\text {reh }}>t_{\text {osc }}$ can also be computed in a similar fashion as above. Since the curvaton energy density is assumed to be negligibly small compared to the total energy at the onset of the oscillation, the curvaton energy continues to be negligible until reheating.

The linear order perturbation is

$$
\frac{\partial \mathcal{N}}{\partial \sigma_{*}}=\frac{r}{4+3 r} \frac{\partial}{\partial \sigma_{*}}\left(\ln \rho_{\sigma \mathrm{osc}}-\ln H_{\mathrm{osc}}^{2}\right),
$$

where the coefficient of $\ln H_{\text {osc }}^{2}$ differs from (2.18) due to different expansions of the universe (i.e. radiation or matter dominated) when the curvaton starts to oscillate.

(2.20) remains the same, hence the second derivative of $\mathcal{N}$ is

$$
\frac{\partial^{2} \mathcal{N}}{\partial \sigma_{*}^{2}}=\frac{16(1+r)}{(4+3 r) r}\left(\frac{\partial \mathcal{N}}{\partial \sigma_{*}}\right)^{2}+\frac{r}{4+3 r} \frac{\partial^{2}}{\partial \sigma_{*}^{2}}\left(\ln \rho_{\sigma \mathrm{osc}}-\ln H_{\mathrm{osc}}^{2}\right) .
$$

\subsection{Curvaton Field Value at the Onset of Oscillation}

In order to evaluate the expressions derived above, we need to know the oscillation amplitude of the curvaton at a certain time, e.g., the curvaton field value at the onset of oscillation $\sigma_{\text {osc }}$. Considering a curvaton rolling along an energy potential $V$ which is a function only of $\sigma$ (hence we do not consider time-dependent potentials, though extending the discussion to such potentials is straightforward), then the curvaton dynamics before it starts its oscillation can be tracked by the attractor solution

$$
c H \dot{\sigma} \simeq-V^{\prime},
$$

where a prime denotes a derivative with respect to $\sigma$, and $c$ is a constant taking the following values depending on the expansion of the universe:

$$
c=\left\{\begin{array}{cl}
3 & \text { (de Sitter) } \\
9 / 2 & \text { (matter domination) } \\
5 & \text { (radiation domination) }
\end{array}\right.
$$

Detailed discussions on this approximation are provided in Appendix A. Here we assume that the approximation (2.24) holds until $t_{\text {osc }}$, when the curvaton suddenly starts a sinusoidal oscillation, cf. Figure 1. Validity of this assumption will be discussed in the next subsection.

Integrating

$$
\frac{d \sigma}{V^{\prime}}=-\frac{d t}{c H}
$$

from the time of horizon exit until the onset of the oscillation, one obtains

$$
\int_{\sigma_{*}}^{\sigma_{\mathrm{osc}}} \frac{d \sigma}{V^{\prime}}=\text { const. }-\int^{H_{\mathrm{osc}}} \frac{d H}{c H \dot{H}},
$$


where $c$ in the right hand side is the one right before $t_{\text {osc }}$, taking either 5 or $9 / 2$ depending on whether $t_{\mathrm{reh}}<t_{\mathrm{osc}}$ or $t_{\mathrm{reh}}>t_{\mathrm{osc}}$, respectively. ${ }^{6}$ We have made use of Assumption 2 upon ignoring the $\sigma_{*}$-dependence of the other terms denoted as const.

Supposing that $H_{\mathrm{osc}}$ is determined merely by $\sigma_{\mathrm{osc}}$, i.e. $H_{\mathrm{osc}}=H_{\mathrm{osc}}\left(\sigma_{\mathrm{osc}}\right)(\mathrm{cf} .(2.33))$, then after differentiating both sides of $(2.27)$ by $\sigma_{*}$, one finds

$$
\frac{\partial \sigma_{\mathrm{osc}}}{\partial \sigma_{*}}=\left\{1-\frac{1}{c(c-3)} \frac{V^{\prime}\left(\sigma_{\mathrm{osc}}\right)}{H_{\mathrm{osc}}^{3}} \frac{\partial H_{\mathrm{osc}}}{\partial \sigma_{\mathrm{osc}}}\right\}^{-1} \frac{V^{\prime}\left(\sigma_{\mathrm{osc}}\right)}{V^{\prime}\left(\sigma_{*}\right)} .
$$

The value of $\sigma_{\text {osc }}$ itself can, in principle, also be computed from (2.27). Taking into account that $H \simeq H_{\mathrm{inf}}=$ const. and $c=3$ during inflation, and that $\dot{H} / H^{2}=3-c$ for matter/radiation domination, one obtains for $t_{\mathrm{reh}}<t_{\mathrm{osc}}$ :

$$
\int_{\sigma_{*}}^{\sigma_{\mathrm{osc}}} \frac{d \sigma}{V^{\prime}}=-\frac{\mathcal{N}_{*}}{3 H_{\mathrm{inf}}^{2}}+\frac{2}{27}\left(\frac{1}{H_{\mathrm{inf}}^{2}}-\frac{1}{H_{\mathrm{reh}}^{2}}\right)+\frac{1}{20}\left(\frac{1}{H_{\mathrm{reh}}^{2}}-\frac{1}{H_{\mathrm{osc}}^{2}}\right),
$$

and for $t_{\mathrm{reh}}>t_{\mathrm{osc}}$ :

$$
\int_{\sigma_{*}}^{\sigma_{\mathrm{osc}}} \frac{d \sigma}{V^{\prime}}=-\frac{\mathcal{N}_{*}}{3 H_{\mathrm{inf}}^{2}}+\frac{2}{27}\left(\frac{1}{H_{\mathrm{inf}}^{2}}-\frac{1}{H_{\mathrm{osc}}^{2}}\right),
$$

where $\mathcal{N}_{*}$ is the number of e-foldings during inflation between the horizon exit of the CMB scale and the end of inflation. Hence for $H_{\mathrm{inf}}^{2} \gg H_{\mathrm{osc}}^{2}$ (and further $H_{\mathrm{reh}}^{2} \gg H_{\mathrm{osc}}^{2}$ for $t_{\mathrm{reh}}<t_{\mathrm{osc}}$ ), we get

$$
\int_{\sigma_{*}}^{\sigma_{\mathrm{osc}}} \frac{d \sigma}{V^{\prime}} \simeq-\frac{\mathcal{N}_{*}}{3 H_{\mathrm{inf}}^{2}}-\frac{1}{2 c(c-3) H_{\mathrm{osc}}^{2}},
$$

where $c$ is the value right before $t_{\mathrm{osc}}$. The two terms in the right hand side can be comparable, e.g., when setting the curvaton (effective) mass squared as $m^{2} \sim H_{\text {inf }}^{2} / 100$ so that the spectral index becomes $\left|n_{s}-1\right| \sim 10^{-2}$, then $H_{\text {osc }}^{2} \sim m^{2} \sim H_{\text {inf }}^{2} / 100 \sim H_{\text {inf }}^{2} / \mathcal{N}_{*}$. Since $H_{\text {osc }}^{2}$ can be given by the curvaton potential as we will soon see, one can derive $\sigma_{\text {osc }}$ as a function of $\sigma_{*}$ by solving (2.31).

\subsection{When the Curvaton Starts Oscillating}

The last piece of information needed to evaluate the density perturbation spectrum is when the curvaton starts its oscillation, i.e., $H_{\mathrm{osc}}$. From the necessary condition (A.5) for the attractor solution (2.24) with (A.6) to hold, one may guess that the oscillation starts when $H^{2}=\left|V^{\prime \prime}\right| / c$. However actually, the curvaton velocity does not increase suddenly at $H^{2}=\left|V^{\prime \prime}\right| / c$, but the curvaton stays on the approximation of the form $H \dot{\sigma} \propto-V^{\prime}$ for some more time. (See also later discussions in Subsection 2.5.) Moreover, the curvaton being on the attractor (2.24) does not necessarily imply that the curvaton oscillation has not yet started.

\footnotetext{
${ }^{6}$ Strictly speaking, here we are further assuming that $c$ stays constant around $t_{\text {osc }}$, so that $c$ is independent of the curvaton field fluctuations. So, e.g., the time of reheating should not be too close to $t_{\mathrm{osc}}$.
} 
Here, instead, we define the onset of the oscillation as when the time scale of the curvaton rolling becomes comparable to the Hubble time, i.e.

$$
\left|\frac{\dot{\sigma}}{H \sigma}\right|_{\mathrm{osc}} \sim 1
$$

where we have assumed that the (local) minimum of the curvaton potential is set to $\sigma=0$. Here it should be noted that modifying (2.32) by constant factors (e.g., choosing the right hand side to be, say, 2) in many cases results merely in modifying $H_{\mathrm{osc}}$ by constant factors, whose contributions to the density perturbations through $\delta \ln H_{\text {osc }}$ drop out, cf. (2.18). Hence throughout this paper we fix the right hand side of the condition (2.32) to be unity.

Then, supposing that the approximation (2.24) holds until $t_{\mathrm{osc}}$ (which is a valid assumption in many cases since, as mentioned above, approximation of the form (2.24) does not break down suddenly at $\left.H^{2}=\left|V^{\prime \prime}\right| / c\right)$, one can rewrite (2.32) as

$$
H_{\mathrm{osc}}^{2}=\left|\frac{V^{\prime}\left(\sigma_{\mathrm{osc}}\right)}{c \sigma_{\mathrm{osc}}}\right|=\frac{V^{\prime}\left(\sigma_{\mathrm{osc}}\right)}{c \sigma_{\mathrm{osc}}} .
$$

Here $c$ is the value right before $t_{\mathrm{osc}}$, and we have removed the absolute value sign in the far right hand side by assuming that the curvaton potential is monotonically increasing (decreasing) for $\sigma>(<) 0$ (otherwise the curvaton following the attractor (2.24) stops rolling before reaching the origin). Thus we obtain

$$
\frac{1}{H_{\mathrm{osc}}} \frac{\partial H_{\mathrm{osc}}}{\partial \sigma_{\mathrm{osc}}}=\frac{1}{2}\left(\frac{V^{\prime \prime}\left(\sigma_{\mathrm{osc}}\right)}{V^{\prime}\left(\sigma_{\mathrm{osc}}\right)}-\frac{1}{\sigma_{\mathrm{osc}}}\right) .
$$

Together with (2.28) this yields

$$
\frac{\partial \sigma_{\mathrm{osc}}}{\partial \sigma_{*}}=\left\{1-\frac{1}{2(c-3)}\left(\frac{\sigma_{\mathrm{osc}} V^{\prime \prime}\left(\sigma_{\mathrm{osc}}\right)}{V^{\prime}\left(\sigma_{\mathrm{osc}}\right)}-1\right)\right\}^{-1} \frac{V^{\prime}\left(\sigma_{\mathrm{osc}}\right)}{V^{\prime}\left(\sigma_{*}\right)} .
$$

\subsection{Results}

Putting together the above results, and also adopting

$$
\rho_{\sigma \mathrm{osc}}=V\left(\sigma_{\mathrm{osc}}\right)
$$

we obtain the final expressions for the density perturbations. Let us define

$$
X\left(\sigma_{\mathrm{osc}}\right) \equiv \frac{1}{2(c-3)}\left(\frac{\sigma_{\mathrm{osc}} V^{\prime \prime}\left(\sigma_{\mathrm{osc}}\right)}{V^{\prime}\left(\sigma_{\mathrm{osc}}\right)}-1\right)
$$

where $c=5$ for $t_{\mathrm{reh}}<t_{\mathrm{osc}}$ and $c=9 / 2$ for $t_{\mathrm{reh}}>t_{\mathrm{osc}}$. Note that $X$ denotes effects due to fluctuations of the time when the curvaton starts its oscillation $\delta H_{\text {osc }}$. 
Then using $\mathcal{P}_{\delta \sigma_{*}}^{1 / 2}=H_{*} / 2 \pi$, the linear order density perturbation spectrum is

$$
\mathcal{P}_{\zeta}=\left(\frac{\partial \mathcal{N}}{\partial \sigma_{*}} \frac{H_{*}}{2 \pi}\right)^{2}
$$

with

$$
\frac{\partial \mathcal{N}}{\partial \sigma_{*}}=\frac{r}{4+3 r}\left(1-X\left(\sigma_{\mathrm{osc}}\right)\right)^{-1}\left\{\frac{V^{\prime}\left(\sigma_{\mathrm{osc}}\right)}{V\left(\sigma_{\mathrm{osc}}\right)}-\frac{3 X\left(\sigma_{\mathrm{osc}}\right)}{\sigma_{\mathrm{osc}}}\right\} \frac{V^{\prime}\left(\sigma_{\mathrm{osc}}\right)}{V^{\prime}\left(\sigma_{*}\right)} .
$$

The second order perturbations in terms of the non-linearity parameter $f_{\mathrm{NL}}$ are

$$
\begin{aligned}
& f_{\mathrm{NL}}= \frac{5}{6} \frac{\partial^{2} \mathcal{N}}{\partial \sigma_{*}^{2}}\left(\frac{\partial \mathcal{N}}{\partial \sigma_{*}}\right)^{-2} \\
&= \frac{40(1+r)}{3 r(4+3 r)}+\frac{5(4+3 r)}{6 r}\left\{\frac{V^{\prime}\left(\sigma_{\mathrm{osc}}\right)}{V\left(\sigma_{\mathrm{osc}}\right)}-\frac{3 X\left(\sigma_{\mathrm{osc}}\right)}{\sigma_{\mathrm{osc}}}\right\}^{-1}\left[\left(1-X\left(\sigma_{\mathrm{osc}}\right)\right)^{-1} X^{\prime}\left(\sigma_{\mathrm{osc}}\right)\right. \\
&+\left\{\frac{V^{\prime}\left(\sigma_{\mathrm{osc}}\right)}{V\left(\sigma_{\mathrm{osc}}\right)}-\frac{3 X\left(\sigma_{\mathrm{osc}}\right)}{\sigma_{\mathrm{osc}}}\right\}^{-1}\left\{\frac{V^{\prime \prime}\left(\sigma_{\mathrm{osc}}\right)}{V\left(\sigma_{\mathrm{osc}}\right)}-\frac{V^{\prime}\left(\sigma_{\mathrm{osc}}\right)^{2}}{V\left(\sigma_{\mathrm{osc}}\right)^{2}}-\frac{3 X^{\prime}\left(\sigma_{\mathrm{osc}}\right)}{\sigma_{\mathrm{osc}}}+\frac{3 X\left(\sigma_{\mathrm{osc}}\right)}{\sigma_{\mathrm{osc}}^{2}}\right\} \\
&\left.+\frac{V^{\prime \prime}\left(\sigma_{\mathrm{osc}}\right)}{V^{\prime}\left(\sigma_{\mathrm{osc}}\right)}-\left(1-X\left(\sigma_{\mathrm{osc}}\right)\right) \frac{V^{\prime \prime}\left(\sigma_{*}\right)}{V^{\prime}\left(\sigma_{\mathrm{osc}}\right)}\right]
\end{aligned}
$$

Thus we have managed to write down curvature perturbations from a curvaton in terms of $\left(r, \sigma_{\text {osc }}, \sigma_{*}\right)$, where $\sigma_{\text {osc }}$ is determined by $\sigma_{*}$ through (2.31).

The curvaton energy fraction upon decay $r(2.17)$ can be estimated by assuming that the universe suddenly switches between matter/radiation domination at reheating (which happens when $H=\Gamma_{\phi}$, with $\Gamma_{\phi}$ the inflaton decay rate) and also when the curvaton dominates the universe (if it ever does),

$$
\begin{aligned}
r=\operatorname{Max} . & {\left[\frac{\rho_{\sigma \mathrm{osc}}}{\rho_{\text {reh }}} \frac{H_{\mathrm{reh}}^{2}}{H_{\mathrm{osc}}^{3 / 2} H_{\mathrm{dec}}^{1 / 2}} \times \operatorname{Min} .\left(1, \frac{H_{\mathrm{reh}}^{1 / 2}}{H_{\mathrm{osc}}^{1 / 2}}\right),\right.} \\
& \left.\left\{\frac{\rho_{\text {o osc }}}{\rho_{\phi \mathrm{reh}}} \frac{H_{\mathrm{reh}}^{2}}{H_{\mathrm{osc}}^{3 / 2} H_{\mathrm{dec}}^{1 / 2}} \times \operatorname{Min} .\left(1, \frac{H_{\mathrm{reh}}^{1 / 2}}{H_{\mathrm{osc}}^{1 / 2}}\right)\right\}^{4 / 3}\right] \\
=\operatorname{Max} . & {\left[\frac{V\left(\sigma_{\mathrm{osc}}\right)}{3 M_{p}^{2} H_{\mathrm{osc}}^{3 / 2} \Gamma_{\sigma}^{1 / 2}} \times \operatorname{Min} .\left(1, \frac{\Gamma_{\phi}^{1 / 2}}{H_{\mathrm{osc}}^{1 / 2}}\right),\right.} \\
& \left.\left\{\frac{V\left(\sigma_{\mathrm{osc}}\right)}{3 M_{p}^{2} H_{\mathrm{osc}}^{3 / 2} \Gamma_{\sigma}^{1 / 2}} \times \operatorname{Min} .\left(1, \frac{\Gamma_{\phi}^{1 / 2}}{H_{\mathrm{osc}}^{1 / 2}}\right)\right\}^{4 / 3}\right],
\end{aligned}
$$

Note that we have again especially made use of Assumption 2. The first and second terms in the Max. parentheses correspond to the curvaton being subdominant and dominant at decay, respectively. Also, the Min. parentheses are due to whether the onset of oscillation is after or before reheating. 


\section{example: Quadratic Potential}

As a simple example, let us consider the familiar case of a quadratic potential $V \propto \sigma^{2}$. One sees that $X$ vanishes, i.e. the onset of the curvaton oscillation is uniform, and

$$
\frac{\partial \mathcal{N}}{\partial \sigma_{*}}=\frac{2 r}{4+3 r} \frac{1}{\sigma_{*}}, \quad f_{\mathrm{NL}}=\frac{5}{12}\left(-3+\frac{4}{r}+\frac{8}{4+3 r}\right),
$$

where the well studied results are reproduced. Here, in particular, large non-Gaussianity $f_{\mathrm{NL}} \gg 1$ is sourced only by curvatons decaying while $r \ll 1$.

\subsection{Validity of the Analytic Expressions}

Upon deriving the above analytic results, we have adopted simplifying assumptions such as sudden decays, and also that the curvaton suddenly starts a sinusoidal oscillation when (2.32). Here we comment on the validity of such assumptions and the analytic results.

First we should remark that upon obtaining the expression (2.33) for $H_{\text {osc }}$, we have assumed the curvaton to follow the attractor solution (2.24) until the onset of the oscillation (2.32). As is discussed in the appendix, this procedure is validated under the necessary condition (A.5). Thus one should be careful when dealing with curvaton potentials along which (A.5) breaks down significantly before the oscillation. There may also be cases where (A.5) once breaks down but recovers afterwards, such that the curvaton once deviates from the approximation (2.24) but comes back again later on, if, say, the curvaton potential possesses a plateau region. However, we also note that the breakdown of (A.5) before the onset of the oscillation does not necessary imply the breakdown of the analytic results derived above. When $\left|V^{\prime \prime} / H^{2}\right|$ becomes of order unity, the parameter $c$ in the approximation (2.24) no longer takes constant values (2.25), but becomes time-dependent as can be seen from (A.4). Hence using the time-dependent solution of (A.4) for $c$ (given that the solution exists ${ }^{7}$ ) may give more accurate estimations, but as long as the time scale of the variation of $c$ is of order the Hubble time, our discussions with constant $c$ should not be drastically modified. Moreover, unless (A.5) breaks down long before the onset of the oscillations, the deviation of the curvaton dynamics from (2.24) with constant $c$ is mild and the above analytic results stay valid. We will see in Subsection 4.1.2 an explicit example where the condition (A.5) clearly breaks down by the time when the oscillation starts, but still the above analytic results giving good estimations of the density perturbations.

Upon estimating $\rho_{\sigma \text { osc }}$, we have adopted (2.36) and ignored the curvaton's kinetic energy. This procedure is validated in many cases where the potential energy is larger than the kinetic energy at the onset of the oscillations. Moreover, as long as the ratio between the potential and kinetic energies is a constant, considerations of the kinetic energy drop out through

\footnotetext{
${ }^{7}$ Note that (A.4) admits solutions of $c$ for largely negative $V^{\prime \prime} / H^{2}$, but has no solutions for largely positive $V^{\prime \prime} / H^{2}$.
} 
$\delta \ln \rho_{\sigma \text { osc }}$. However, there may be cases for which it becomes important to take into account the curvaton's kinetic energy at $t_{\text {osc }}$.

It was also assumed that even if the curvaton potential deviates from a quadratic one (giving rise to $\delta H_{\mathrm{osc}}$ ), once the oscillation starts its amplitude soon decays and the curvaton starts a sinusoidal oscillation about its minimum where the potential is well approximated by a quadratic one. However, we should also note that if the curvaton oscillation starts with an amplitude large enough so that its potential deviates from quadratic for a while after $t_{\mathrm{osc}}$, then the curvaton energy density does not behave as matter but decays as $\rho_{\sigma} \propto a^{-n}$ with $n \neq 3$. This can modify our analytic expressions, in addition to changing $r$ and thus the amplitude of the perturbation spectrum. In cases where the oscillation history is rather

trivial (e.g. $V=\frac{1}{2} m^{2} \sigma^{2}+\lambda \sigma^{4}$ giving $n=4 \rightarrow n=3$ ), extending the discussions above is rather straightforward, as is shown in Appendix B. However for more complicated cases (e.g. curvaton oscillation in a potential with superimposed periodic modulations, which can give rise to a time-dependent $n$ ), some parts of our results may require major corrections. We leave this for future work.

\subsection{Spectral Index}

Although the perturbation amplitude crucially depends on the curvaton dynamics after inflation, the spectral index

$$
n_{s}-1 \equiv \frac{d \ln \mathcal{P}_{\zeta}}{d \ln k}
$$

is determined only by the information at the horizon exit during inflation. Note that the linear order density perturbation is expressed by

$$
\frac{\partial \mathcal{N}}{\partial \sigma_{*}}=\left(\text { function of } r, \sigma_{\mathrm{osc}}\right) \times \frac{1}{V^{\prime}\left(\sigma_{*}\right)},
$$

where $r$ and $\sigma_{\text {osc }}$ are independent of the comoving wave number $k$. Hence

$$
n_{s}-1=\frac{d}{d \ln k} \ln \left(\frac{H_{*}}{V^{\prime}\left(\sigma_{*}\right)}\right)^{2} \simeq \frac{2}{3} \frac{V^{\prime \prime}\left(\sigma_{*}\right)}{H_{*}^{2}}+2 \frac{\dot{H}_{*}}{H_{*}^{2}},
$$

where we have made use of $d \ln k \simeq H_{*} d t$ and $3 H_{*} \dot{\sigma}_{*} \simeq-V^{\prime}\left(\sigma_{*}\right)$. One clearly sees that for inflation with $H_{*}=$ const., the observationally suggested red-tilted perturbation spectrum requires the curvaton to be tachyonic during inflation. See also discussions in Footnote 4.

\section{Curvatons Along Flat Potentials}

The results (2.39) and (2.40) in the previous section show that additional contributions to the density perturbations from $\delta H_{\text {osc }}$ can suppress/enhance the perturbation amplitude as well as the non-Gaussianity. As an interesting case where the $\delta H_{\mathrm{osc}}$ contributions to the density 
perturbations become important, in this section we focus on a curvaton starting its oscillation from a flat region where the potential tilt is tiny.

Given that the first derivative of the potential at the onset of oscillation is small to enough to satisfy

$$
\left|\frac{\sigma_{\mathrm{osc}} V^{\prime \prime}\left(\sigma_{\mathrm{osc}}\right)}{V^{\prime}\left(\sigma_{\mathrm{osc}}\right)}\right|,\left|\frac{V^{\prime \prime}\left(\sigma_{\mathrm{osc}}\right) V\left(\sigma_{\mathrm{osc}}\right)}{V^{\prime}\left(\sigma_{\mathrm{osc}}\right)^{2}}\right|,\left|\frac{V^{\prime \prime}\left(\sigma_{\mathrm{osc}}\right)^{2}}{V^{\prime}\left(\sigma_{\mathrm{osc}}\right) V^{\prime \prime \prime}\left(\sigma_{\mathrm{osc}}\right)}\right|,\left|\frac{\sigma_{\mathrm{osc}} V^{\prime \prime}\left(\sigma_{*}\right)}{V^{\prime}\left(\sigma_{\mathrm{osc}}\right)}\right| \gg 1,
$$

then one finds

$$
\left|X\left(\sigma_{\mathrm{osc}}\right)\right| \simeq\left|\frac{1}{2(c-3)} \frac{\sigma_{\mathrm{osc}} V^{\prime \prime}\left(\sigma_{\mathrm{osc}}\right)}{V^{\prime}\left(\sigma_{\mathrm{osc}}\right)}\right| \gg 1, \quad X^{\prime}\left(\sigma_{\mathrm{osc}}\right) \simeq-\frac{1}{2(c-3)} \frac{\sigma_{\mathrm{osc}} V^{\prime \prime}\left(\sigma_{\mathrm{osc}}\right)^{2}}{V^{\prime}\left(\sigma_{\mathrm{osc}}\right)^{2}},
$$

and the equations (2.39) and (2.40) are approximated by

$$
\begin{gathered}
\frac{\partial \mathcal{N}}{\partial \sigma_{*}} \simeq \frac{3 r}{4+3 r} \frac{V^{\prime}\left(\sigma_{\mathrm{osc}}\right)}{\sigma_{\mathrm{osc}} V^{\prime}\left(\sigma_{*}\right)}, \\
f_{\mathrm{NL}} \simeq-\frac{5(4+3 r)}{18 r} \frac{\sigma_{\mathrm{osc}} V^{\prime \prime}\left(\sigma_{*}\right)}{V^{\prime}\left(\sigma_{\mathrm{osc}}\right)} .
\end{gathered}
$$

Note that here, $\left|f_{\mathrm{NL}}\right| \gg 1$ is guaranteed (even when $r \gg 1$ ). Furthermore, when $V(\sigma)$ is a monotonically increasing function around its origin, i.e. $V^{\prime}>(<) 0$ for $\sigma>(<) 0$, then $f_{\mathrm{NL}} \propto$ (negative factor) $\times V^{\prime \prime}\left(\sigma_{*}\right)$. In other words, with constant $H$ inflation, a red(blue)-tilted perturbation spectrum is always accompanied by a largely positive (negative) $f_{\mathrm{NL}}$.

We should remark that under the condition $\left|\sigma V^{\prime \prime} / V^{\prime}\right| \gg 1$ in (3.1), (A.5) no longer holds at $t_{\text {osc }}$. This may lead to some deviation of the curvaton dynamics from the approximation (2.24), giving rise to errors in the estimations. However, as we will see in Section 4 when we study explicit cases, the overall behaviour of the resulting density perturbations discussed here are still valid as long as the breakdown of the approximation (2.24) is mild. (See also discussions in Subsection 2.5).

\subsection{Hilltop Curvatons}

Let us further focus on a curvaton located at the hilltop, i.e. a curvaton whose potential is well approximated by

$$
V(\sigma)=V_{0}-\frac{1}{2} m^{2}\left(\sigma-\sigma_{0}\right)^{2}
$$

around $\sigma_{\text {osc }}$ and $\sigma_{*}$, where $m, \sigma_{0}$, and $V_{0}(>0)$ are constants. Such case will be important when we study pseudo-Nambu-Goldstone curvatons in the next section. Without loss of generality, hereafter we assume $0<\sigma_{\text {osc }}<\sigma_{*}<\sigma_{0}$.

Now the flatness conditions in (3.1) translate into

$$
\frac{\sigma_{\mathrm{osc}}}{\sigma_{0}-\sigma_{\mathrm{osc}}} \gg 1, \quad \frac{V_{0}}{m^{2}\left(\sigma_{\mathrm{osc}}-\sigma_{0}\right)^{2}} \gg 1,
$$


under which (3.3) and (3.4) are

$$
\begin{aligned}
\mathcal{P}_{\zeta}^{1 / 2} & \simeq \frac{3 r}{4+3 r} \frac{\sigma_{0}-\sigma_{\mathrm{osc}}}{\sigma_{0}-\sigma_{*}} \frac{H_{\mathrm{inf}}}{2 \pi \sigma_{\mathrm{osc}}} \\
f_{\mathrm{NL}} & \simeq \frac{5(4+3 r)}{18 r} \frac{\sigma_{\mathrm{osc}}}{\sigma_{0}-\sigma_{\mathrm{osc}}}
\end{aligned}
$$

with spectral index

$$
n_{s}-1=-\frac{2}{3} \frac{m^{2}}{H_{\mathrm{inf}}^{2}}<0 .
$$

Here we note that throughout this subsection, inflation with constant $H$ is assumed.

The Hubble parameter at the onset of oscillation (2.33) is

$$
H_{\mathrm{osc}}^{2}=\frac{m^{2}\left(\sigma_{0}-\sigma_{\mathrm{osc}}\right)}{c \sigma_{\mathrm{osc}}} \ll H_{\mathrm{inf}}^{2},
$$

where the inequality follows from (3.6) and $\left|n_{s}-1\right|=\mathcal{O}\left(10^{-2}\right)$. Thus $\sigma_{*}$ and $\sigma_{\text {osc }}$ can be related by solving (2.31), which in the hilltop limit, i.e. $\sigma_{*} \rightarrow \sigma_{0}$ (from below), gives

$$
\ln \left(\frac{\sigma_{0}-\sigma_{*}}{\sigma_{0}-\sigma_{\mathrm{osc}}}\right) \simeq-\frac{1}{2(c-3)} \frac{\sigma_{\mathrm{osc}}}{\sigma_{0}-\sigma_{\mathrm{osc}}},
$$

where $c=5$ for $t_{\mathrm{reh}}<t_{\mathrm{osc}}$ and $c=9 / 2$ for $t_{\mathrm{reh}}>t_{\mathrm{osc}}$. Since the left hand side is logarithmic, one sees that $\sigma_{\text {osc }}$ approaches $\sigma_{0}$ much slower than $\sigma_{*}$ does, cf. Figure 2. Thus, in the hilltop limit, $\mathcal{P}_{\zeta}(3.7)$ blows up due to the enhancement factor $\left(\sigma_{0}-\sigma_{\text {osc }}\right) /\left(\sigma_{0}-\sigma_{*}\right)$, while $f_{\mathrm{NL}}$ (3.8) increases slowly. One can see from this example that the evaluation of $\sigma_{\text {osc }}$ is crucial for correctly estimating the density perturbations. The extreme amplification of the perturbation amplitude can be understood as curvatons taking longer time to start its oscillation as one approaches the hilltop limit.

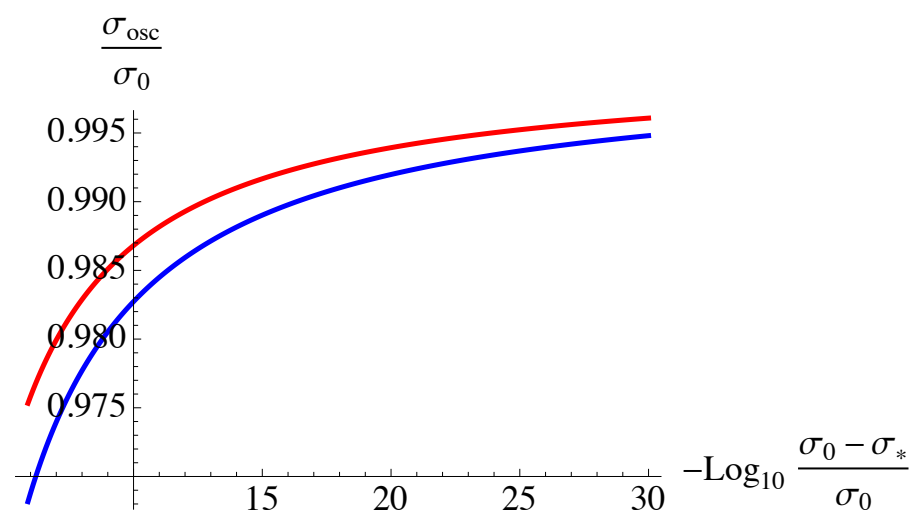

Figure 2: $\sigma_{\text {osc }}$ in terms of $\sigma_{*}$, obtained from (3.11) with $c=5$ (red) and $c=9 / 2$ (blue). 
One may worry, in the hilltop limit, that the curvaton during inflation jumps over the hill due to quantum fluctuations $\Delta \sigma= \pm H_{\text {inf }} / 2 \pi$, and domain walls be generated. Here we note that if the hilltop curvaton generates density perturbations that satisfy the observational constraints from the WMAP 7 -year data [16] $\mathcal{P}_{\zeta} \approx 2.4 \times 10^{-9}$ and $f_{\mathrm{NL}}<74$ (for local-type non-Gaussianity which is important for us here), the condition for the absence of domain walls is satisfied,

$$
\frac{H_{\mathrm{inf}}}{2 \pi}\left(\sigma_{0}-\sigma_{*}\right)^{-1} \simeq \frac{6}{5} \mathcal{P}_{\zeta}^{1 / 2} f_{\mathrm{NL}} \lesssim 0.004
$$

We also have to check whether quantum fluctuations lead to a random walk of the curvaton. Further using the WMAP7 central value $n_{s} \approx 0.96$, one sees that the classical rolling dominates over the quantum fluctuations,

$$
\frac{H_{\mathrm{inf}}}{2 \pi}\left|\frac{\dot{\sigma}_{*}}{H_{\mathrm{inf}}}\right|^{-1}=\frac{3}{2 \pi} \frac{H_{\mathrm{inf}}^{3}}{V^{\prime}\left(\sigma_{*}\right)} \simeq \frac{12}{5} \frac{\mathcal{P}_{\zeta}^{1 / 2} f_{\mathrm{NL}}}{1-n_{s}} \lesssim 0.2 .
$$

Since the onset of the curvaton oscillation is delayed as one approaches the hilltop limit, it becomes easier for the curvaton to dominate the universe before it decays. However, we should also note that the curvaton energy fraction when it starts to oscillate,

$$
\frac{\rho_{\sigma \mathrm{osc}}}{3 M_{p}^{2} H_{\mathrm{osc}}^{2}} \simeq \frac{3 r}{4+3 r} \frac{4 c}{5} \frac{f_{\mathrm{NL}}}{1-n_{s}} \frac{V_{0}}{3 M_{p}^{2} H_{\mathrm{inf}}^{2}} \lesssim \frac{3 r}{4+3 r} \frac{V_{0}}{3 M_{p}^{2} H_{\mathrm{inf}}^{2}} \times 7000,
$$

can become as large as unity. In such case, the above estimations can break down since upon deriving the analytical expressions, we have ignored the effects of the curvaton energy density on the evolution of the universe before the curvaton starts its oscillation, cf. Assumption 2.

\section{Case Study: Pseudo-Nambu-Goldstone Curvatons}

As a simple example of a curvaton model, let us examine the case where the curvaton is a pseudo-Nambu-Goldstone (NG) boson of a broken U(1) symmetry. The approximate symmetry suppresses the curvaton mass, while the periodicity of the $\mathrm{U}(1)$ provides both (local) minima and maxima along the curvaton potential, thus allowing the curvaton to produce a blue-tilted as well as a red-tilted density perturbation spectrum.

In particular, we study the NG curvaton potential of the form

$$
V(\sigma)=\Lambda^{4}\left[1-\cos \left(\frac{\sigma}{f}\right)\right]
$$

where $f$ and $\Lambda$ are mass scales. Without loss of generality, we set the field value of the curvaton at horizon exit during inflation to lie within the range $0<\sigma_{*}<\pi f$, and consider its oscillation about the origin $\sigma=0$. Supposing that the coupling of the NG curvaton with its decay product is suppressed by the scale of symmetry breaking $f$, then the decay rate of the curvaton takes the value

$$
\Gamma_{\sigma}=\frac{\beta}{16 \pi} \frac{m^{3}}{f^{2}}=\frac{\beta}{16 \pi} \frac{\Lambda^{6}}{f^{5}}
$$


where $m$ is the mass at the minimum, i.e. $m^{2}=V^{\prime \prime}(0)$, and $\beta$ is a constant whose precise value depends on the decay modes. Throughout this section we fix $\beta$ to unity. (Cases with smaller $\beta$ will be discussed towards the end of Subsection 4.2.2). Furthermore, we consider inflation with constant $H$, and assume the pivot scale to have exited the horizon 50 e-foldings before the end of inflation.

We will see that an NG curvaton away from the hilltop requires inflation and the reheating scales to lie within a narrow range in order to generate density perturbations with spectral index of order $n_{s}-1 \sim-10^{-2}$. However, we also show that as one approaches the hilltop limit $\sigma_{*} \rightarrow \pi f$, the allowed inflation/reheating scales extremely broaden and that the nonGaussianity lies in the range $10 \lesssim f_{\mathrm{NL}} \lesssim 30$.

For an investigation of NG curvatons located close to their potential minimum, see also [23].

\subsection{Density Perturbations from NG Curvatons}

Let us first investigate density perturbations generated by NG curvatons, and compare the analytic estimations in the previous sections with numerically computed results. We start from NG curvatons located away from the hilltop of the potential, then move on to the hilltop limit.

\subsubsection{Non-Hilltop Region}

Firstly, we choose the potential parameters and the inflation/reheating scales such that the curvaton with $\sigma_{*}=\frac{3}{4} \pi f$ generates a density perturbation spectrum whose amplitude takes the COBE normalization value

$$
\mathcal{P}_{\zeta} \approx 2.4 \times 10^{-9}
$$

with the spectral index lying at the central value of the WMAP7 [16] bound (for a power-law spectrum with no tensor modes)

$$
n_{s} \approx 0.96
$$

Then, considering the case where $t_{\mathrm{osc}}<t_{\mathrm{reh}}$, and that the curvaton dominates the universe before it decays, we adopt the following parameter set (which lies at around the center of the allowed window in Figure 11 in the next subsection): $H_{\text {inf }}=10^{13.5} \mathrm{GeV}, \rho_{\text {reh }}^{1 / 4}=10^{15} \mathrm{GeV}$ (i.e. $\left.\Gamma_{\phi} \approx 9.73 \times 10^{-8} M_{p}\right), f \approx 3.36 \times 10^{-2} M_{p}$, and $\Lambda \approx 3.56 \times 10^{-4} M_{p}$.

In order to analytically estimate the density perturbations, we need to solve (2.31) for obtaining $\sigma_{\text {osc }}$. Introducing

$$
\alpha \equiv \frac{\sigma}{\pi f},
$$

one obtains

$$
\ln \left|\frac{\tan \left(\alpha_{\mathrm{osc}} \pi / 2\right)}{\tan \left(\alpha_{*} \pi / 2\right)}\right|=-\frac{\mathcal{N}_{*}}{3 H_{\mathrm{inf}}^{2}} \frac{\Lambda^{4}}{f^{2}}-\frac{1}{2(c-3)} \frac{\alpha_{\mathrm{osc}} \pi}{\sin \left(\alpha_{\mathrm{osc}} \pi\right)},
$$


whose solution for $\mathcal{N}_{*}=50$ and $c=9 / 2$ in the region $0.01 \lesssim \alpha_{*} \lesssim 0.99$ is approximated by (cf. Figure 3)

$$
\alpha_{\mathrm{osc}} \approx 0.195 \alpha_{*}-0.0993 \alpha_{*}^{2}+0.846 \alpha_{*}^{4}-1.34 \alpha_{*}^{6}+1.26 \alpha_{*}^{8} .
$$

Then by plugging (2.41), (4.1), and (4.7) into (2.39) and (2.40), one obtains the linear order density perturbations and its non-Gaussianity as functions of $\alpha_{*}=\sigma_{*} / \pi f$.

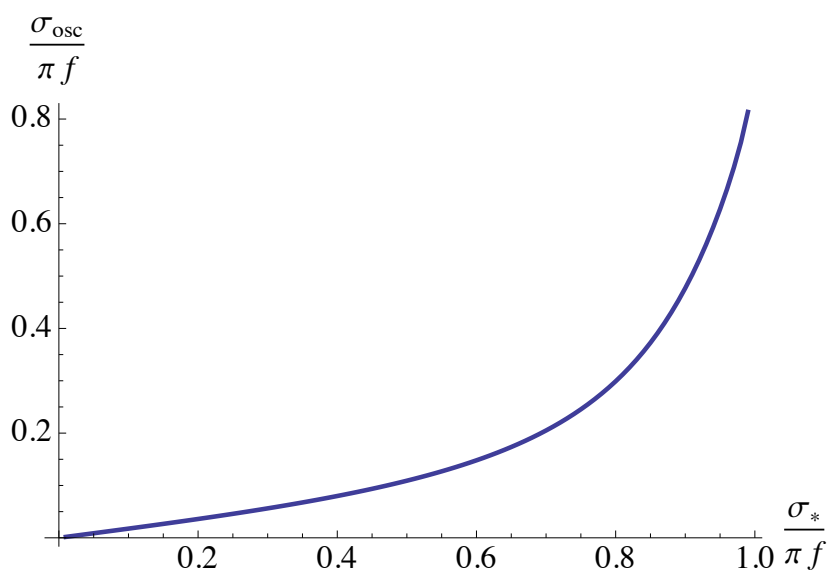

Figure 3: $\sigma_{\text {osc }}$ in terms of $\sigma_{*}$ for the NG curvaton, cf. (4.7).

We have also numerically computed the density perturbations by solving the equation of motion of the curvaton. After 50 e-foldings of inflation with constant $H$ since the exit of the pivot scale, the inflaton energy is transferred to inflaton matter decaying as $\rho_{m} \propto a^{-3}$, which eventually decays into radiation $\left(\rho_{r} \propto a^{-4}\right)$. Sudden decays are adopted for the inflaton and the curvaton, i.e. they instantly decay into radiation when $H=\Gamma$.

We run the numerical computations with different $\sigma_{*}$ (the curvaton velocity at the horizon exit is set to the slow-roll attractor value, which is uniquely determined by $\sigma_{*}$ ), and evaluate the number of e-foldings obtained by the time the curvaton decays. Thus evaluating the differences in e-folding numbers $\Delta \mathcal{N}$ due to shifts in the initial condition $\Delta \sigma$, we numerically obtain $\partial \mathcal{N} / \partial \sigma_{*}$. Second order perturbations as well as the spectral index are computed in similar manners.

In Figures 4 - 7 we lay out the results in terms of $\sigma_{*}$, where the solid lines are the analytic estimations and the dots are the numerical results. Blue lines and dots correspond to our case with $\Gamma_{\sigma}=\frac{1}{16 \pi} \frac{m^{3}}{f^{2}}$ where the WMAP values (4.3) and (4.4) are realized at $\alpha_{*}=3 / 4$, and for comparison we also show cases with $\Gamma_{\sigma}=10^{4} \times \frac{1}{16 \pi} \frac{m^{3}}{f^{2}}$ (red) and $\Gamma_{\sigma}=10^{-4} \times \frac{1}{16 \pi} \frac{m^{3}}{f^{2}}$ (green). (Note that Figure 4 has only one line since the spectral index is independent of the decay rate.) One sees that the results asymptote to that of the familiar quadratic curvaton (2.42) as $\alpha_{*} \rightarrow 0$. On the other hand, the linear order perturbation as well as the non-Gaussianity 
increases as one approaches $\alpha_{*} \rightarrow 1$, smoothly connecting to the hilltop limit which we will soon study. The curvaton energy fraction at decay $r$ increases with $\alpha_{*}$, and the density perturbations become $r$-independent for $r \gg 1$, as can be seen from the overlap of the blue and green lines towards $\alpha_{*} \rightarrow 1$.

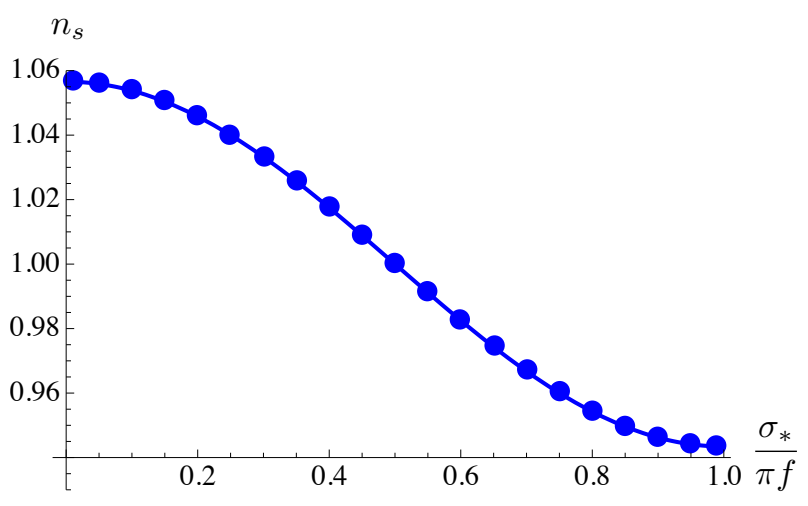

Figure 4: Spectral index.

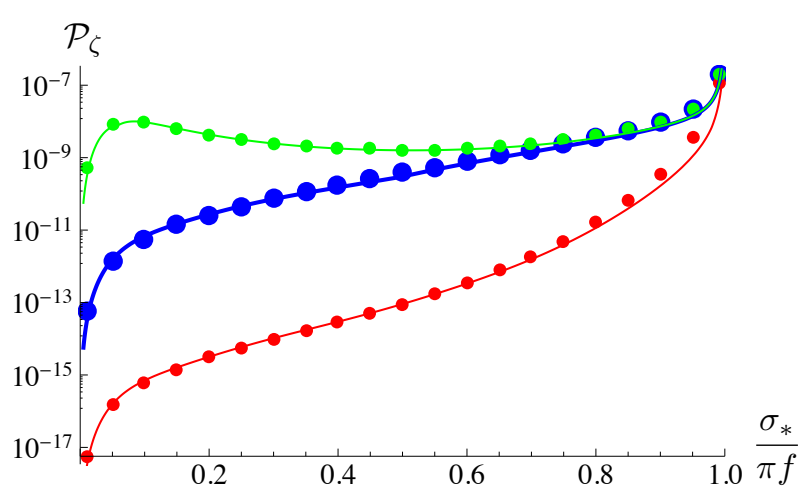

Figure 6: Power spectrum.

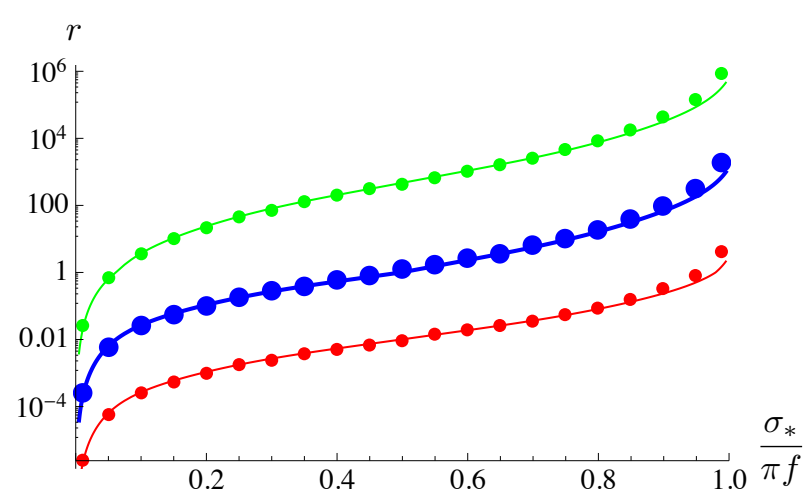

Figure 5: Energy fraction at decay.

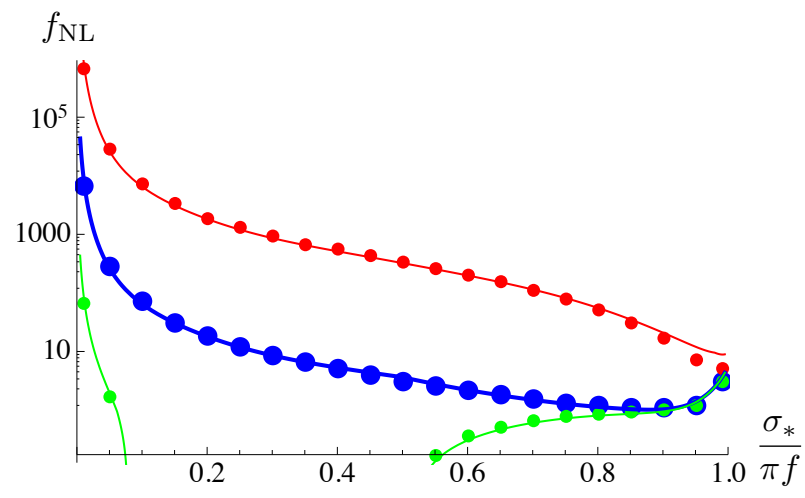

Figure 7: Non-Gaussianity. (The green line goes negative in the covered region.)

\subsubsection{Hilltop Region}

As we have seen in Subsection 3.1, an extreme enhancement of the density perturbations occurs in the hilltop region. Let us now choose parameters such that density perturbations with (4.3) and (4.4) are realized at $\sigma_{*}=\left(1-10^{-8}\right) \pi f$. Considering the case where $t_{\text {osc }}<t_{\text {reh }}$ and the curvaton dominates the universe before it decays, we adopt the following parameter set (which lies at around the center of the allowed window in Figure 15): $H_{\text {inf }}=10^{6} \mathrm{GeV}$, $\rho_{\text {reh }}^{1 / 4}=10^{9} \mathrm{GeV}\left(\right.$ i.e. $\Gamma_{\phi} \approx 9.73 \times 10^{-20} M_{p}$ ), $f \approx 1.57 \times 10^{-3} M_{p}$, and $\Lambda \approx 1.23 \times 10^{-8} M_{p}$. 
Introducing $n_{*}$ as

$$
\alpha_{*} \equiv 1-10^{-n_{*}}
$$

the solution of (4.6) in the region of our interest $2 \lesssim n_{*} \lesssim 10$ is approximated by

$$
\alpha_{\mathrm{osc}} \approx 1-\left(0.416+0.0120 n_{*}\right) n_{*}^{-1.47}
$$

which shows that $\alpha_{\text {osc }}$ approaches unity much slower than $\alpha_{*}$ does (cf. Figure 2). Then one can evaluate the density perturbation spectrum as was done for the non-hilltop region. The results are shown in Figures 8 - 10, where again the solid lines denote the analytic estimations and dots the numerically computed results. The curvaton well dominates the universe before it decays, i.e. $r \gg 1$, in the displayed region $5 \lesssim n_{*} \lesssim 9$. Here we should remark that, in the hilltop limit, the attractor (2.24) (mildly) breaks down before (2.32) is realized, hence $\sigma_{\text {osc }}(4.9)$ estimated from (2.33) contains error of order $\Delta \sigma_{\text {osc }} / \sigma_{\text {osc }}=\mathcal{O}(1){ }^{8}$ This gives rise to errors of the similar order in the analytic estimations, which is clearly seen in Figure 10. Therefore, we have also calculated semi-analytic results by substituting numerically computed $\sigma_{\text {osc }}$ (defined by (2.32)) into the analytic expressions in Subsection 2.4. The semi-analytic results are shown in the figures as dashed lines, which match well with the numerical computations. (The dashed line is absent in Figure 8 since the spectral index is evaluated independently of $\left.\sigma_{\text {osc }}\right)$.

The figures show that, as was discussed as generic features of hilltop curvatons in Subsection 3.1, the linear order density perturbations blow up in the hilltop limit, while the non-linearity parameter $f_{\mathrm{NL}}$ increases slowly. The spectral index, since it is determined by the potential curvature, asymptotes to a constant value as one approaches the hilltop. However in the extreme hilltop limit the finite size field fluctuations $\delta \sigma_{*}=H_{\text {inf }} / 2 \pi$ can no longer be treated as infinitesimal, giving rise to the tiny variations of the numerical results seen in Figure 8.

\subsection{Parameter Space}

Now that we have studied the behaviour of density perturbations from NG curvatons, let us explore the parameter space which allows generation of observationally consistent density perturbations. Out of the five free parameters $\left(f, \Lambda, H_{\mathrm{inf}}, H_{\mathrm{reh}}, \sigma_{*}\right)$, two are fixed from the COBE normalization (4.3) and the WMAP7 central value for the spectral index (4.4). We then seek constraints on the inflation/reheating scales under various $\sigma_{*}$, and discuss cosmology with NG curvatons.

\footnotetext{
${ }^{8}$ The value of $-V^{\prime} / H \dot{\sigma}$ (which in the analytic estimations we have treated as a constant $c=9 / 2$ ) becomes as large as $\sim 30$ right before the onset of the oscillation (2.32). (This can be estimated by solving the equation (A.4) in terms of $c$.) Nevertheless, the error in $\sigma_{\text {osc }}$ is of order unity since $-V^{\prime} / H \dot{\sigma}$ being large is limited to times close to $t_{\mathrm{osc}}$.
} 


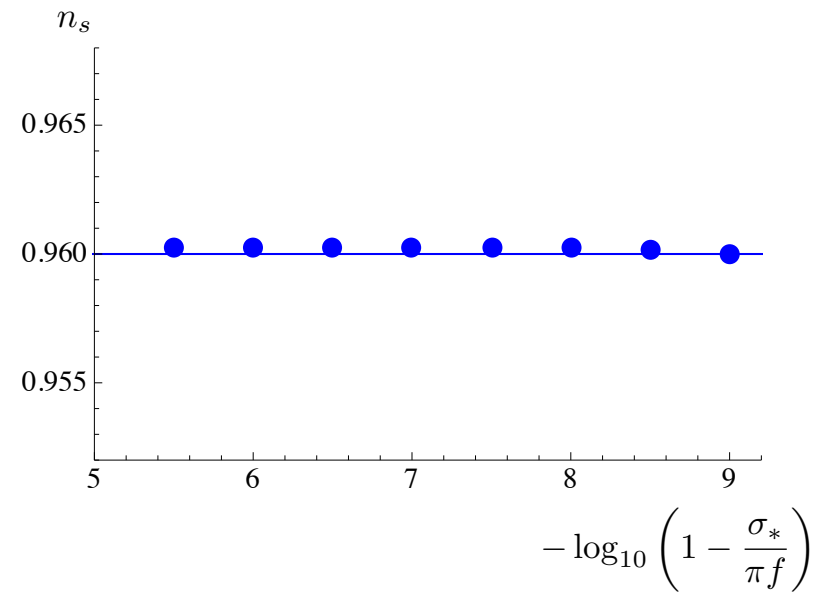

Figure 8: Spectral index.

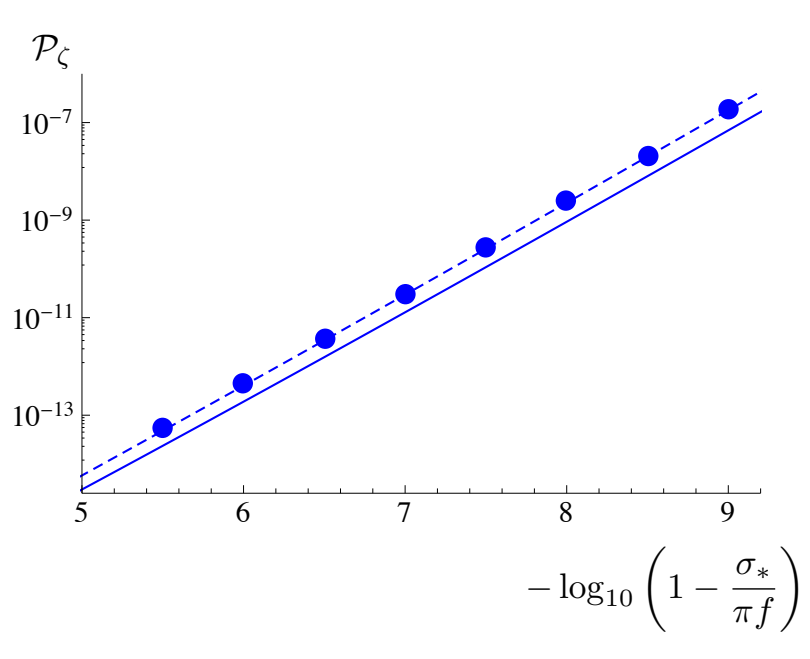

Figure 9: Power spectrum.

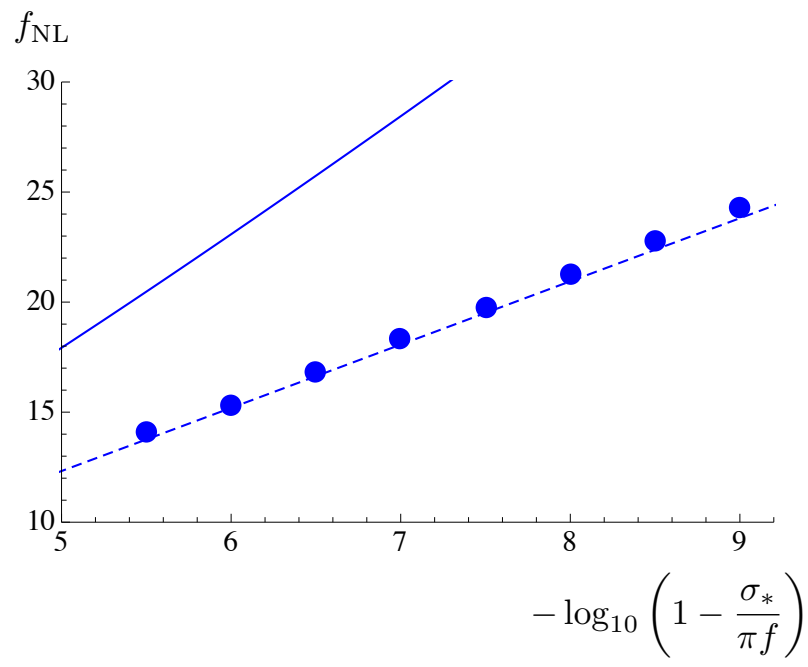

Figure 10: Non-Gaussianity. 


\subsubsection{Requirements}

Let us begin by laying out the requirements for a consistent curvaton scenario. Firstly, we assume the curvaton energy density to be negligibly small during inflation,

$$
\frac{V\left(\sigma_{*}\right)}{3 M_{p}^{2} H_{\mathrm{inf}}^{2}} \ll 1,
$$

and until the curvaton starts its oscillation (cf. Assumption 2),

$$
\frac{V\left(\sigma_{\mathrm{osc}}\right)}{3 M_{p}^{2} H_{\mathrm{osc}}^{2}} \ll 1 .
$$

Also, quantum fluctuations during inflation should not make the curvaton jump over its potential minimum in order to avoid the resulting density perturbations from being highly non-Gaussian, or over the maximum to avoid domain walls,

$$
\frac{H_{\mathrm{inf}}}{2 \pi} \ll \sigma_{*} \ll \pi f-\frac{H_{\mathrm{inf}}}{2 \pi} .
$$

We further require the curvaton's classical rolling to dominate over the quantum fluctuations during inflation (so that we can have definite predictions for the perturbation spectrum ${ }^{9}$ ),

$$
\frac{3}{2 \pi} \frac{H_{\mathrm{inf}}^{3}}{V^{\prime}\left(\sigma_{*}\right)} \ll 1,
$$

where the slow-roll approximation for the curvaton during inflation is verified by (4.10) and (4.19). The curvaton decay should happen after reheating

$$
\Gamma_{\phi}>\Gamma_{\sigma}
$$

and also after the onset of the curvaton oscillation

$$
H_{\mathrm{osc}}>\Gamma_{\sigma},
$$

but at the latest before the Big Bang Nucleosynthesis $(\mathrm{BBN})$ at temperature $T_{\mathrm{BBN}} \sim 1 \mathrm{MeV}$ $[30,31,32,33]$,

$$
3 M_{p}^{2} \Gamma_{\sigma}^{2}>(1 \mathrm{MeV})^{4}
$$

We also require the oscillating curvaton's mass to be larger than its decay temperature, in order to avoid possible backreaction effects to the curvaton's perturbative decay (see e.g. $[34,35,36]$ for discussions on this issue). Assuming instant thermalization, this condition is written roughly as

$$
V^{\prime \prime}(0)>\left(3 M_{p}^{2} \Gamma_{\sigma}^{2}\right)^{1 / 2}
$$

\footnotetext{
${ }^{9}$ The randomized case of NG curvatons are discussed in [23].
} 
The resulting density perturbations should satisfy the COBE normalization,

$$
\mathcal{P}_{\zeta} \approx 2.4 \times 10^{-9},
$$

and for its spectral index we adopt the WMAP7 central value (4.4), which through (2.45) translates into (note that we are supposing inflation with $H=$ const.)

$$
\frac{2}{3} \frac{V^{\prime \prime}\left(\sigma_{*}\right)}{H_{\mathrm{inf}}^{2}} \approx-0.04 .
$$

We note that when (4.19) is satisfied at $\sigma_{*} \approx \pi f / 2$ with $\left|\cos \left(\sigma_{*} / f\right)\right| \ll 1$, then a rather large $\Lambda^{4} / f^{2}$ can give rise to a large running of the spectral index and may contradict with observations. However, when fixing $n_{s}-1 \sim-10^{-2}$, the allowed parameter window becomes small anyway as $\sigma_{*}$ approaches the inflection point $\pi f / 2$ (as we will soon see), thus we merely require (4.19) for the spectral tilt. Bounds on local-type non-Gaussianity from the WMAP7 (at $95 \% \mathrm{CL}$ ) are

$$
-10<f_{\mathrm{NL}}<74 .
$$

Constraints on primordial gravitational waves set an upper bound on the inflationary energy scale. The 7 -year $\mathrm{WMAP}+\mathrm{BAO}+H_{0}$ gives $\mathcal{P}_{T} / \mathcal{P}_{\zeta}<0.24$ (95\% CL), i.e.,

$$
H_{\text {inf }}<1.3 \times 10^{14} \mathrm{GeV} \text {. }
$$

However, we note that when the inflation scale is high enough to (come close to) saturate the bound (4.21), then depending on the inflationary mechanism, one can expect to have a contribution to the spectral index from a non-vanishing $\dot{H} / H^{2}$ (see also Footnote 4), as well as the central value of the spectral index bounds (4.4) being slightly shifted (to about 0.97 with the 7 -year $\left.\mathrm{WMAP}+\mathrm{BAO}+H_{0}\right)$. Here, as we have stated at the beginning of this subsection, we adopt the value (4.4) and a $H=$ const. inflation, and simply use (4.21) as an upper bound on the inflationary scale.

Finally, reheating, i.e. inflaton decay, should happen subsequent to inflation,

$$
\Gamma_{\phi}<H_{\text {inf }}
$$

\subsubsection{Windows for Inflation/Reheating Scales}

Among the above constraints, (4.18) and (4.19) can be used to fix the parameters $f$ and $\Lambda$. Here, note that $\sigma_{\text {osc }} / f$ is estimated as a function of $\sigma_{*} / f$ by solving (4.6) under $\mathcal{N}_{*}=50$, setting $c=5($ or $9 / 2)$ for $t_{\mathrm{osc}}>($ or $<) t_{\mathrm{reh}}$, and choosing $\Lambda^{4} / f^{2} H_{\mathrm{inf}}^{2}$ as a function of $\sigma_{*} / f$ from (4.19).

Thus we obtain windows in the parameter space that satisfy all the above constraints (4.10) - (4.22) in four regimes, categorized by $r \gtrless 1$ and $t_{\text {osc }} \gtrless t_{\text {reh }}$. The results are displayed in Figures $11-16$, in the $H_{\text {inf }}-\rho_{\text {reh }}^{1 / 4}$ plane under various values of $\sigma_{*} / f$. The allowed windows are shown as colored regions, where the yellow (green) regions correspond to that for $r>1$ 
with $t_{\mathrm{osc}}>t_{\mathrm{reh}}\left(t_{\mathrm{osc}}<t_{\mathrm{reh}}\right)$, and the blue (red) regions for $r<1$ with $t_{\mathrm{osc}}>t_{\mathrm{reh}}\left(t_{\mathrm{osc}}<t_{\mathrm{reh}}\right)$, respectively. The right side edge of each box, i.e. the maximum displayed value of $H_{\text {inf }}$, is set by the gravitational wave bound (4.21), also the gray shaded region is excluded since the inflaton should decay after inflation (4.22). Differently colored boundaries of the allowed regions denote different constraints being saturated, where purple dashed: curvaton being subdominant at the onset of oscillation (4.11), green: curvaton decay after reheating (4.14), orange dashed: BBN (4.16), and red: upper bound on $f_{\mathrm{NL}}$ in (4.20). Furthermore, the blue lines denote where $r=1$. The inequalities $\ll$ and $\gg$ in the above conditions are relaxed to $<$ and $>$ upon obtaining the allowed windows in the figures, hence in this sense the displayed constraints are conservative bounds. ${ }^{10}$ Moreover, we note that we have examined the constraints independently in four distinct regions $t_{\mathrm{osc}} \gg t_{\mathrm{reh}}\left(\ll t_{\mathrm{reh}}\right)$ and $r \gg 1(\ll 1)$, and displayed (two of) them together in each figure. This is why the displayed boundaries bend sharply when crossing $t_{\mathrm{osc}}=t_{\mathrm{reh}}$ and moving to differently colored regions. However, we expect that such simplifications and also errors due to approximations used in the analytic analyses (including that upon estimating $\sigma_{\mathrm{osc}}$ in the hilltop limit, see discussions below (4.9)) are minor for obtaining order-of-magnitude estimations of the inflation/reheating scales compatible with NG curvatons. We also show contour lines of the curvaton decay temperature for $r>1$ cases, where the numbers in boxes denote $\left(3 M_{p}^{2} \Gamma_{\sigma}^{2}\right)^{1 / 4}[\mathrm{GeV}]$. (Note that the contour lines are actually relevant only on the colored allowed regions.)

First of all, requiring a red-tilted density perturbation spectrum, the NG curvaton at horizon exit should be located at $1 / 2<\alpha_{*}=\sigma_{*} / \pi f<1$. Constraints on NG curvatons away from the hilltop can be seen in Figures 11 and 12, where the representative value $\alpha_{*}=3 / 4$ is taken. The dominant and subdominant cases share the same regions in the $H_{\text {inf }}-\rho_{\text {inf }}^{1 / 4}$ plane, bounded by (4.21) and (4.22). Here, one sees that NG curvatons away from the hilltop require rather high inflation/reheating scales. This is because the spectral index $\left|n_{s}-1\right| \sim 10^{-2}$ indicates a curvaton's effective mass as large as $\left|m_{\text {eff }}\right|^{2} \sim 10^{-2} H_{\text {inf }}^{2}$, which starts the curvaton oscillation soon after inflation ends. ${ }^{11}$ Unless the inflation and reheating scales are high enough to provide a sufficiently long radiation-dominated era for the oscillating curvaton to dominate the universe, the curvaton cannot source substantial density perturbations. This is especially the case as one approaches the inflection point of the potential, i.e. $\alpha_{*} \rightarrow 1 / 2$, since then $\left|\cos \left(\sigma_{*} / f\right)\right| \rightarrow 0$ and the tilt of the potential increases, pushing $H_{\text {osc }}(2.33)$ as well as the inflation/reheating scales even higher.

On the other hand, as one approaches the hilltop limit $\alpha_{*} \rightarrow 1$, the onset of the oscillation is delayed and curvaton domination is allowed with lower inflation/reheating scales. Moreover,

\footnotetext{
${ }^{10}$ Even under the relaxed conditions, we especially note that $\frac{V\left(\sigma_{\mathrm{osc}}\right)}{3 M_{p}^{2} H_{\mathrm{osc}}^{2}}<r$ is guaranteed by the conditions (4.14), (4.15), and the analytic expression for $r$ (2.41).

${ }^{11}$ Strictly speaking, as we have emphasized in the previous sections, it is not the potential's curvature but rather its tilt that determines the onset of the oscillation. However, away from the inflection point $\sigma / \pi f=1 / 2$ or the hilltop $\sigma / \pi f=1$, the curvature and tilt are of the same scale, i.e. $\left|V^{\prime \prime}\right| \sim V^{\prime} / \sigma$, hence either can be used for estimating $H_{\mathrm{osc}}$.
} 
the linear order density perturbations obtain an enhancement factor $\left(1-\alpha_{\text {osc }}\right) /\left(1-\alpha_{*}\right)$, which should be compensated by a smaller $H_{\text {inf }} / f$ (cf. (3.7)). This translates into a larger $\Lambda$ through the fixed spectral index (4.19), thus further increases the curvaton energy density fraction, cf. (3.14). Figure 13 with $\alpha_{*}=1-10^{-4}$ shows that lower inflation/reheating scales are compatible with hilltop NG curvatons, thus broadening the allowed window. One also sees that now our requirement of the curvaton being subdominant until the onset of oscillation (4.11) becomes important, cutting off the right edge (i.e. high inflation scales).

As we have noted in Subsections 3.1 and 4.1.2, non-Gaussianity also increases, though mildly, towards the hilltop. In the allowed region in Figure 13, the non-Gaussianity is $f_{\mathrm{NL}} \sim 10$, except for close to the left edge where $r \sim 1$ and hence larger $f_{\mathrm{NL}}$. (Here, upon evaluating the explicit value of $f_{\mathrm{NL}}$, we have carried out numerical calculations in order to avoid errors rising from the approximations used in the analytic estimations.) For a subdominant curvaton whose non-Gaussianity is further enhanced, the observational upper bound on $f_{\mathrm{NL}}(4.20)$ becomes a severe constraint as is seen in Figure 14. Eventually the allowed window for subdominant curvatons vanishes as one approaches the hilltop and the non-Gaussianity increases. When $\log _{10}\left(1-\alpha_{*}\right)$ is about -8 , the window exists only for dominant curvatons, which is shown in Figure 15. Here, the left edge is now the non-Gaussianity bound.

Further approaching the tip, the BBN constraint (4.16) kicks in from low $H_{\text {inf }}$, cf. Figure 16 where $\alpha_{*}=1-10^{-11}$. The non-Gaussianity in most of the allowed region of Figure 16 (except for close to the bottom edge) is $f_{\mathrm{NL}} \sim 30$. As one goes even closer to the hilltop, the BBN constraint goes towards higher $H_{\text {inf }}$ while the constraint (4.11) goes towards lower $H_{\text {inf }}$, thus removes the allowed window completely by $\log _{10}\left(1-\alpha_{*}\right) \sim-12$.

When the curvaton decay rate is further suppressed, i.e. when $\beta$ in (4.2) is way smaller than unity, the overall behaviour of the allowed window stays the same except that the curvaton decaying temperature is lowered correspondingly. For instance, when $\beta=10^{-4}$, the blue lines in the figures where $r=1$ are shifted towards lower $H_{\text {inf }}$ by about one order of magnitude (or towards lower $\rho_{\text {inf }}^{1 / 4}$ by about two orders). The red lines $\left(f_{\mathrm{NL}}\right.$ bound) shift similarly while the purple dashed lines (4.11) are independent of $\beta$. Therefore the allowed windows for dominant curvatons slightly broaden in Figures 11, 13, 15, while the windows for subdominant curvatons simply shift towards lower $H_{\mathrm{inf}}, \rho_{\mathrm{inf}}^{1 / 4}$. However, since the BBN constraint becomes severer for lower curvaton decay temperatures, the allowed window for dominant curvatons vanishes at $\log _{10}\left(1-\alpha_{*}\right) \sim-10$.

Let us comment on implications of the present scenario for the origin of the baryon asymmetry and dark matter. Focusing on the case of the dominant curvaton, the adiabatic density perturbation becomes fixed when the curvaton dominates the universe. In order to avoid generating too large isocurvature perturbations, therefore, the baryon as well as dark matter abundance must be fixed after that. The thermal leptogenesis scenario [37] requires a relatively high curvaton decay temperature $T_{\text {dec }} \gtrsim 10^{9} \mathrm{GeV}$. In the present scenario, $T_{\text {dec }}$ exceeds $10^{9} \mathrm{GeV}$ for $1-\alpha_{*} \gtrsim 10^{-3}$. As $\alpha_{*}$ further approaches 1 , the decay temperature decreases and other baryogenesis scenarios are needed. For instance, non-thermal leptogenesis [38] is known 
to work for $T_{\text {dec }} \gtrsim 10^{6} \mathrm{GeV}$, which will be viable for $1-\alpha_{*} \gtrsim 10^{-5}$. For the Affleck-Dine mechanism $[39,40]$ to work, the following condition must be met, $H_{\text {dom }}>m_{\mathrm{AD}}$, where $H_{\text {dom }}$ denotes the Hubble parameter when the curvaton dominates the universe, and $m_{\mathrm{AD}}\left(\gtrsim m_{3 / 2}\right)$ denotes the mass of the Affleck-Dine field. The above condition can be met for various $\alpha_{*}$ as long as the reheating scale is not so low. The electroweak baryogenesis requires the curvaton decay temperature to exceed of order $100 \mathrm{GeV}$, which is translated into $1-\alpha_{*} \gtrsim 10^{-8}$. For $1-\alpha_{*} \lesssim 10^{-8}$, the decay of $\sigma$ may be able to generate the baryon asymmetry in a way similar to Ref. [41], using the R-parity violating operators. On the other hand, there are many dark matter candidates whose abundance is fixed after the curvaton dominates the universe. For instance, the (non-)thermal relic of the WIMPs and the QCD axion would fit with the present scenario.

Summarizing, the allowed parameter window for NG curvatons in the $H_{\text {inf }}-\rho_{\text {inf }}^{1 / 4}$ plane broadens significantly in the hilltop regime, especially for $1-10^{-4} \lesssim \sigma_{*} / \pi f \lesssim 1-10^{-11}$. In such hilltop regime, the NG curvaton mainly dominates the universe before it decays, but still produces non-Gaussianity in the density perturbations of size $10 \lesssim f_{\mathrm{NL}} \lesssim 30$. We reiterate that this prediction on $f_{\mathrm{NL}}$ is free from the errors due to approximations used for the analytic estimations, although the constraints displayed in the figures contain errors corresponding to order unity factors.

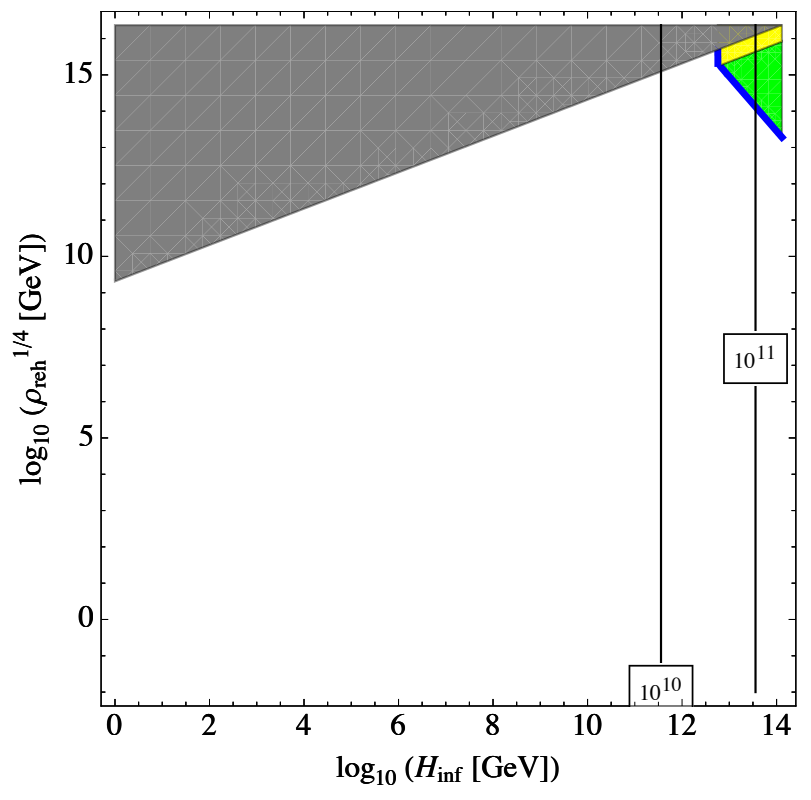

Figure 11: $\sigma_{*} / \pi f=3 / 4, r>1$.

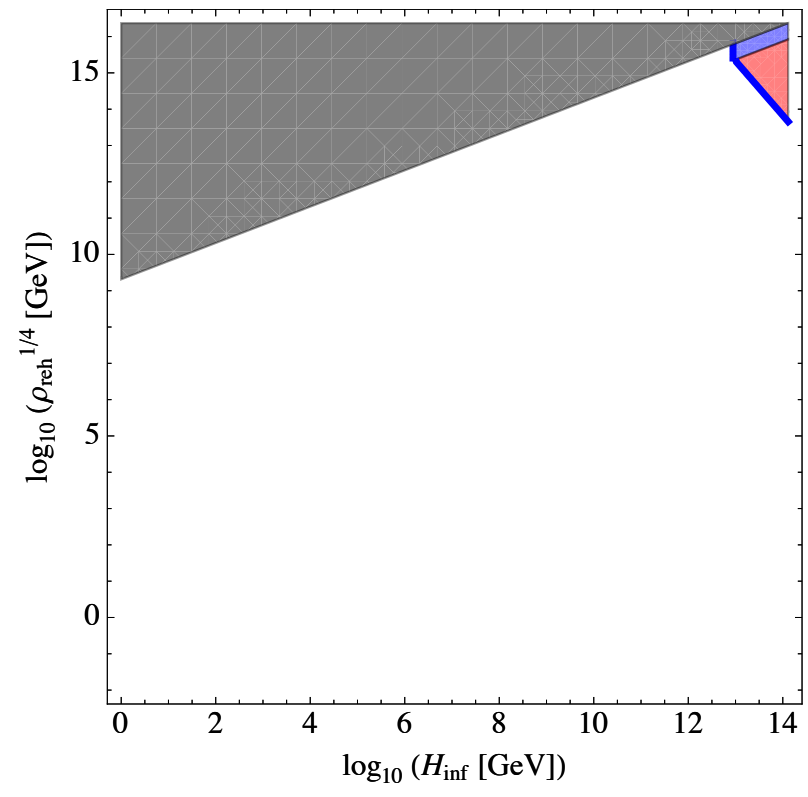

Figure 12: $\sigma_{*} / \pi f=3 / 4, r<1$. 

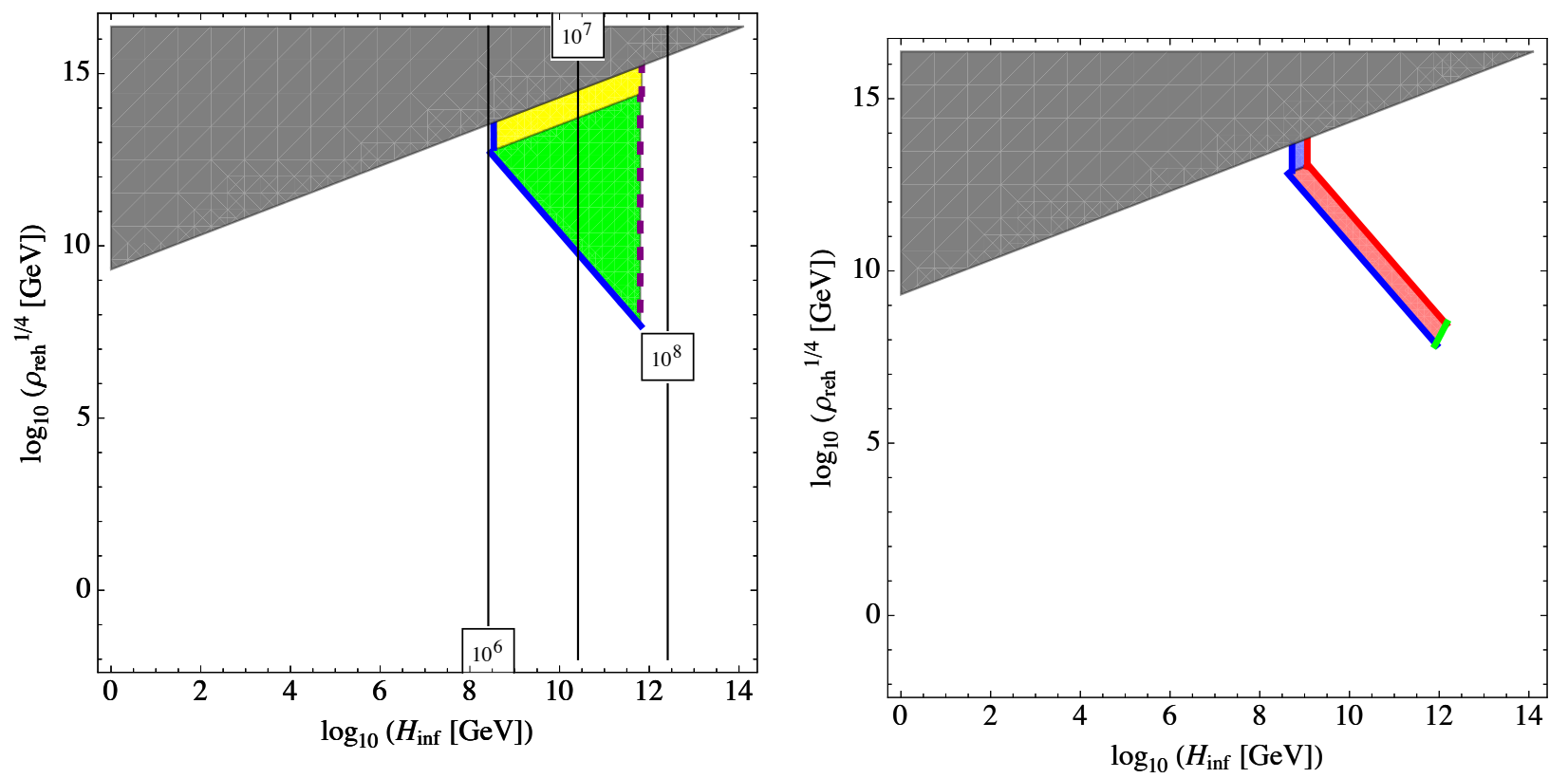

Figure 13: $\sigma_{*} / \pi f=1-10^{-4}, r>1$. $f_{\mathrm{NL}} \sim 10$ in most of the allowed region.
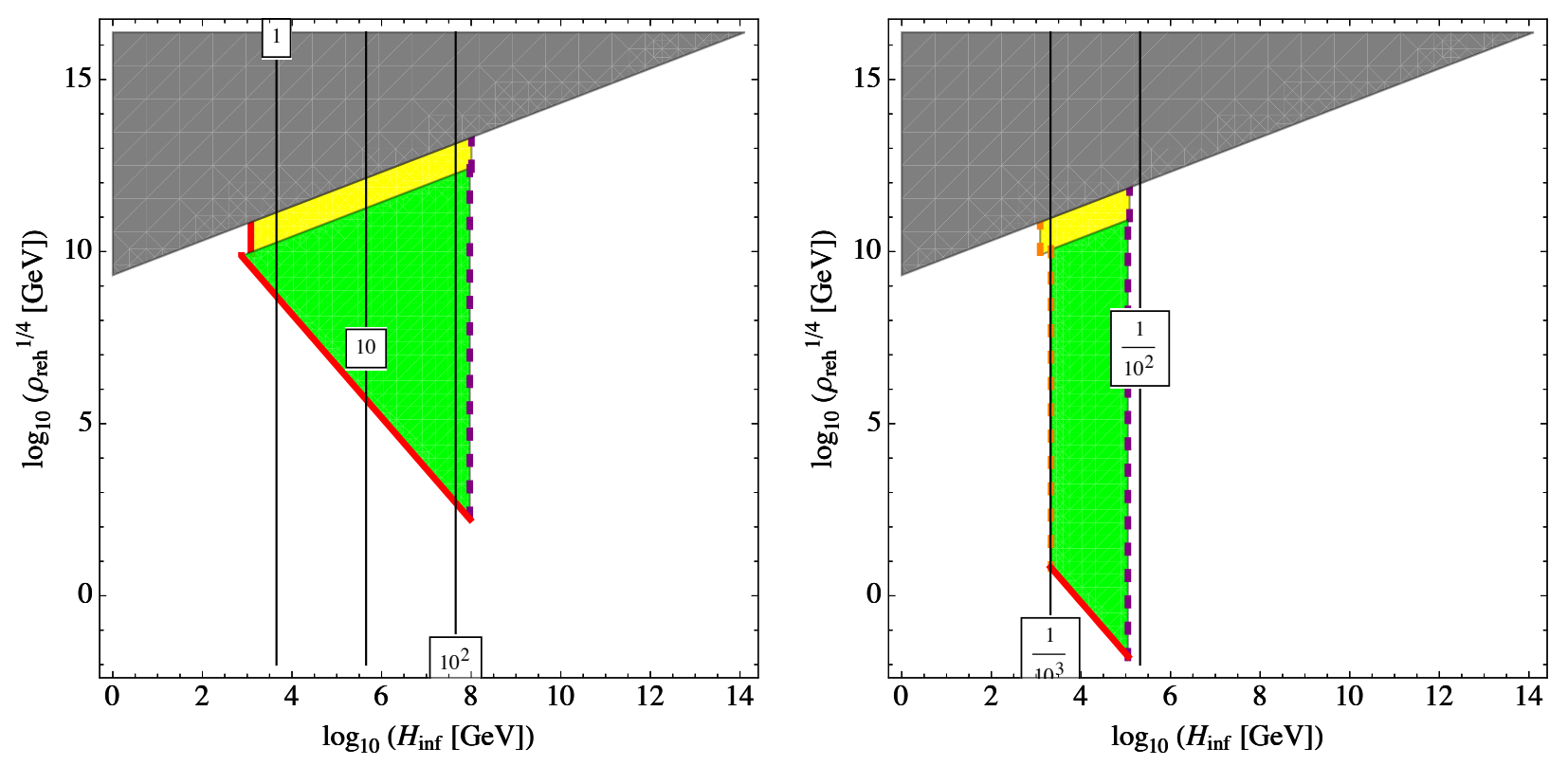

Figure 15: $\sigma_{*} / \pi f=1-10^{-8}, r>1$. Figure 16: $\sigma_{*} / \pi f=1-10^{-11}, r>1$. $f_{\mathrm{NL}} \sim 20$ in most of the allowed region. $f_{\mathrm{NL}} \sim 30$ in most of the allowed region. 


\subsection{Discussion on Hilltop Curvatons}

Let us comment on initial conditions of hilltop curvaton models. So far we have simply assumed that the curvaton sits near the local maximum. This may be justified if the curvaton is charged under some symmetry, which is unbroken during early stage of the inflation or preinflationary epoch. This ameliorates the amount of fine-tuning of the initial condition for the hilltop curvaton. On the other hand, in the NG curvaton model, there is no special point in its field space since the curvaton has an approximate shift symmetry. Then, a priori, there is no particular reason to believe that the curvaton initially sits near the local maximum. However, we do not know whether the conventional naturalness argument can be really applied to the determination of parameters in the early universe. For example, the parameters may be determined in a similar fashion as in the anthropic argument for explaining the present value of the cosmological constant [42]. Suppose that the prior probability of the initial position of the NG curvaton has a flat distribution in the field space. Assuming that all the other parameters are fixed, it is conceivable that only the universe with the curvaton initially sitting near the local maximum is anthropically selected, if it does not lead to the habitable universe otherwise. For instance, this is expected to be the case, if the inflaton mainly decays into the hidden sector while the curvaton decays into the standard model particles. Thus, the apparent fine-tuning of the initial condition of the NG curvaton based on the conventional naturalness argument may be due to our ignorance of the mechanism about how those parameters are determined.

Another important constraint on the curvaton model is that it must account for the red-tilted power spectrum, $n_{s}-1 \sim-10^{-2}$. As long as the curvaton potential is timeindependent, this implies that the curvature of the curvaton potential is only about one order of magnitude smaller than the Hubble parameter during inflation. Then, unless the curvaton is initially sitting near the hilltop, it starts to oscillate about the potential minimum soon after inflation, and the curvaton mechanism does not work for most inflation models. Thus we are led to consider the hilltop curvaton models. However, the situation changes if we allow the curvaton potential to depend on time. In particular, the curvaton may have a negative Hubble mass during inflation, $V \supset-\kappa H^{2} \sigma^{2}$. Then the red-tilted spectrum simply implies $\kappa \sim 10^{-2}$. After the inflaton decays, the negative Hubble mass term disappears, and the curvaton potential may become very flat, and the curvaton starts to oscillate long afterward. In this case we expect that the curvaton scenario works for many inflation models while accounting for $n_{s}-1 \sim-10^{-2} .{ }^{12}$

\footnotetext{
${ }^{12}$ We note however that the curvaton dynamics would be complicated with a negative Hubble mass term. For instance, the curvaton slow-rolls on the negative Hubble mass away from its origin during inflation, then long after reheating, the curvaton comes back and starts to oscillate about the origin. Alternatively, the redtilted power spectrum can be easily realized in the curvaton model with a global U(1) symmetry; if the radial component of the complex scalar field moves from a large field value to a smaller one, the phase component (i.e. NG curvaton) would have a scale-dependent fluctuation, which results in the red-tilted power spectrum. MK and FT thank Shinta Kasuya for discussion on this issue.
} 
Lastly we comment on a possible reason why the NG curvaton has a mass close to the Hubble parameter during inflation. If the curvaton potential is generated by non-perturbative dynamics, e.g. strong gauge interactions, the potential height is expected to be determined by the dynamical scale, and it is exponentially sensitive to the value of the gauge coupling at the UV scale. So, if there is a bias toward a larger value of the coupling constant at the UV scale, the curvaton mass is favored to be as large as possible. If the curvaton mass exceeds the Hubble parameter during inflation, it is stabilized at the potential minimum, and the curvaton mechanism does not work. Thus, the curvaton mass slightly smaller than $H_{\text {inf }}$ may be selected in the curvaton landscape. Of course this is just a theoretical possibility, and it is hard to prove that such an argument is indeed the correct explanation.

\section{Conclusions}

In this paper, we have explored density perturbations from a curvaton with a generic potential, and shown that the curvaton's field fluctuations can lead to non-uniform onset of the curvaton oscillation $\delta H_{\text {osc }}$. This sources additional contributions to the resulting density perturbations, in addition to that from the field fluctuations directly perturbing the curvaton energy density. We showed that the combination of the two effects gives rise to non-trivial behaviours for density perturbations from curvatons, such as the curvaton sourcing large $f_{\mathrm{NL}}$ with either sign even if it dominates the universe before decay. The effect due to $\delta H_{\text {osc }}$ becomes important when the curvaton potential deviates from the familiar quadratic type. Since a curvaton potential with negative curvature is required during inflation in order to explain the observationally suggested red-tilted density perturbation spectrum (without invoking inflation with large $\left|\dot{H} / H^{2}\right|$ ), it is of importance to evaluate effects from $\delta H_{\text {osc }}$. Our work was also motivated by curvaton models based on microscopic physics such as supersymmetry or string theory, which provides explicit examples of curvaton potentials possessing non-trivial forms.

As a simple case where the $\delta H_{\text {osc }}$ effects become dominant on the generated density perturbations, we have studied curvatons that start their oscillations from a flat region along its potential. Especially for hilltop curvatons, we showed the strong enhancement of the linear order density perturbations $\mathcal{P}_{\zeta}$, accompanied by a mild increase of the non-Gaussianity $f_{\mathrm{NL}}$.

Another non-trivial case, which we have not studied intensively in this paper, is when various contributions to the density perturbations cancel each other and thus suppress the perturbation amplitude. Conversely, then the non-Gaussianity is expected to become large. It would be interesting to investigate such cases, which may be realized by, e.g., curvaton potentials possessing plateau regions, or with superimposed repeated modulations. ${ }^{13}$ We leave this for future work.

\footnotetext{
${ }^{13}$ Superimposed repeated modulations to the inflaton potential can also have interesting consequences to the density perturbation spectrum generated by the inflaton itself, see e.g. [43, 44, 45, 46, 47, 48, 49, 50, 51, 52].
} 
We also applied our generic results to study a simple model where the curvaton is a pseudo-Nambu-Goldstone (NG) boson of a broken U(1) symmetry. Although NG curvatons away from the hilltop require high inflation/reheating scales in order to generate red-tilted density perturbation spectra with $n_{s}-1 \sim-10^{-2}$, it was shown that NG curvatons at the hilltop can work with a wide range of inflation/reheating scales. We also showed that working NG curvatons in the hilltop predict the non-Gaussianity to lie in the range $10 \lesssim f_{\mathrm{NL}} \lesssim 30$, which is accessible to upcoming CMB observations such as the PLANCK satellite.

For the four-point correlation functions of the density perturbations from hilltop NG curvatons, one obtains relations that are similar to those for the familiar quadratic curvatons, i.e., $\tau_{\mathrm{NL}} \sim g_{\mathrm{NL}} \sim f_{\mathrm{NL}}^{2}$. However, different curvaton potentials may lead to different behaviours among the non-Gaussianities (see related discussions in [12]). It will be interesting to examine systematically the range of possibilities arising from curvatons with generic energy potentials.

Both from observational and theoretical reasons, it is important to investigate in detail the conversion process of the curvaton field fluctuations into the resulting density perturbations. In this work, we have provided generic analytic expressions incorporating effects due to nonuniform onset of the curvaton oscillation, which open up new possibilities for the curvaton paradigm.

\section{Acknowledgements}

FT thanks Tomo Takahashi for useful communication on the non-uniform onset of oscillations in the curvaton scenario. This work was supported by the Grant-in-Aid for Scientific Research on Innovative Areas (No. 21111006) [FT,MK], Scientific Research (A) (No. 22244030 and 21244033 [FT]), Scientific Research (C) (No. 22540267 [MK]) and JSPS Grant-in-Aid for Young Scientists (B) (No. 21740160) [FT]. This work was also supported by World Premier International Center Initiative (WPI Program), MEXT, Japan.

\section{A Scalar Field Dynamics in an Expanding Universe}

In this appendix we discuss dynamics of a scalar field $\sigma$ rolling along a potential $V(\sigma)$ in an expanding universe background. Here the potential $V(\sigma)$ is assumed to be a function only of $\sigma$, and does not depend on say, time, explicitly. We especially show a condition under which the equation of motion of the field

$$
\ddot{\sigma}+3 H(t) \dot{\sigma}+\frac{\partial V(\sigma)}{\partial \sigma}=0
$$

is approximated by

$$
c H(t) \dot{\sigma} \simeq-\frac{\partial V(\sigma)}{\partial \sigma}
$$

where $c$ is a constant. 
The background expansion is considered to be independent of the $\sigma$-dynamics (as in the case of a curvaton before it starts to oscillate), and the universe to be dominated by some sort of energy density with equation of state $p=w \rho$ (with constant $w$ ), so that the Hubble parameter satisfies

$$
\frac{\dot{H}}{H^{2}}=-\frac{3(w+1)}{2} .
$$

By taking a time derivative of both sides of (A.2) and comparing with (A.1), one can check that

$$
c \simeq 3-\frac{\dot{H}}{H^{2}}-\frac{V^{\prime \prime}}{c H^{2}}=3+\frac{3(w+1)}{2}-\frac{V^{\prime \prime}}{c H^{2}},
$$

where a prime denotes a derivative with respect to $\sigma$. Hence for

$$
\left|\frac{V^{\prime \prime}}{c H^{2}}\right| \ll 1,
$$

we obtain

$$
c=3+\frac{3(w+1)}{2}
$$

which is no less than 3 for $w \geq-1$. For example, $c=3$ for a de Sitter, $c=9 / 2$ for a matter dominated, and $c=5$ for a radiation dominated universe. (We note that a non-zero but constant $\left|V^{\prime \prime}\right| / H^{2}$ can also realize the approximation (A.2) with different values of $c$, as in rapid-roll inflation $[53,54,55,56,57,58,59]$.

One can further show that (A.2) is an attractor. Introducing

$$
\xi \equiv \frac{\ddot{\sigma}+3 H \dot{\sigma}}{c H \dot{\sigma}}-1
$$

where we now define $c$ by (A.6), then one can check that

$$
\frac{\dot{\xi}}{H}=-\frac{V^{\prime \prime}}{c H^{2}}-c \xi-c \xi^{2} .
$$

Since $c$ given by (A.6) is positive, this equation shows that, at least when $|\xi|<1,|\xi|$ damps as the universe expands until its amplitude becomes as small as $\mathcal{O}\left(\left|V^{\prime \prime}\right| / c^{2} H^{2}\right)$. Hence as long as the condition (A.5) holds, the approximation (A.2) with (A.6) is a stable attractor.

\section{B Density Perturbations from Curvatons with Non- Sinusoidal Oscillations}

In this appendix we generalize the expressions for density perturbations in the main body of the paper to incorporate a period of non-sinusoidal curvaton oscillation during which the energy density redshifts as $\rho_{\sigma} \propto a^{-n}$ with arbitrary $n$, followed by the usual sinusoidal oscillation with $n=3$. The results here can be applied to, e.g., a curvaton potential which is 
quadratic around its (local) minimum, but is dominated by a non-quadratic polynomial term at large $\sigma$.

Instead of the assumptions in Subsection 2.1, here we suppose that at some time $t_{\mathrm{osc}}$ after inflation the curvaton starts an oscillation which redshifts away its energy density as $\rho_{\sigma} \propto a^{-n}$ with an arbitrary constant $n$, until its energy density becomes as small as $\rho_{\sigma}=\rho_{\sigma \sin }$, then the curvaton suddenly switches to a sinusoidal oscillation giving $\rho_{\sigma} \propto a^{-3}$. After some period of the sinusoidal oscillation, the curvaton decays to radiation. Having in mind potentials that switch between quadratic and non-quadratic polynomials at a certain field value, we take the energy density $\rho_{\sigma \sin }$ as a constant which is independent of $\sigma_{*}$. We also consider the curvaton energy density fraction to be negligibly small until $t_{\text {reh }}$ or $t_{\mathrm{osc}}$, whichever is later.

Then one can compute the $\delta \mathcal{N}$ similarly as in Subsection 2.1, yielding

$$
\begin{gathered}
\frac{\partial \mathcal{N}}{\partial \sigma_{*}}=\frac{r}{4+3 r} \frac{\partial}{\partial \sigma_{*}}\left(\frac{3}{n} \ln \rho_{\sigma \mathrm{osc}}-A \ln H_{\mathrm{osc}}^{2}\right) \\
\frac{\partial^{2} \mathcal{N}}{\partial \sigma_{*}^{2}}=\frac{16(1+r)}{(4+3 r) r}\left(\frac{\partial \mathcal{N}}{\partial \sigma_{*}}\right)^{2}+\frac{r}{4+3 r} \frac{\partial^{2}}{\partial \sigma_{*}^{2}}\left(\frac{3}{n} \ln \rho_{\sigma \mathrm{osc}}-A \ln H_{\mathrm{osc}}^{2}\right),
\end{gathered}
$$

where $A=3 / 4$ for $t_{\mathrm{reh}}<t_{\mathrm{osc}}$, and $A=1$ for $t_{\mathrm{reh}}>t_{\mathrm{osc}}$ (in this latter case $t_{\mathrm{reh}}$ can be either before or after $t_{\sin }$, but of course before $t_{\mathrm{dec}}$ ). We note that $(2.20)$ holds in the present case as well. It is clear that the above expressions reproduce those in Subsection 2.1 for $n=3$. Moreover, the discussions in Subsections 2.2 and 2.3 can be applied to the present case without any modification.

\section{example: Linear Curvatons}

As an example for applying the above formulae, let us take a look at a curvaton potential of the form

$$
V(\sigma)=\Lambda^{4}\left(\frac{\sigma}{f}\right)^{2}\left[1+\left(\frac{\sigma}{f}\right)^{2}\right]^{-1 / 2},
$$

with constant mass scales $\Lambda$ and $f$. The potential is quadratic around its minimum $\sigma=0$, but approaches a linear potential at $|\sigma| \gg f$.

Considering the curvaton to start its oscillation in the linear regime, i.e. $\sigma_{\text {osc }} \gg f$, then by solving (2.31), one finds

$$
\sigma_{\mathrm{osc}}=\frac{2 c-6}{2 c-5}\left(\sigma_{*}-\frac{\mathcal{N}_{*}}{3} \frac{\Lambda^{4}}{f H_{\mathrm{inf}}^{2}}\right) .
$$

The energy density of the curvaton oscillating along the linear regime redshifts as $\rho_{\sigma} \propto a^{-2}$, hence one can use the formulae (B.1) and (B.2) to obtain

$$
\frac{\partial \mathcal{N}}{\partial \sigma_{*}}=\frac{(3+2 A) r}{2(4+3 r)}\left(\sigma_{*}-\frac{\mathcal{N}_{*}}{3} \frac{\Lambda^{4}}{f H_{\mathrm{inf}}^{2}}\right)^{-1},
$$




$$
f_{\mathrm{NL}}=\frac{5}{6 r}\left\{\frac{16(1+r)}{4+3 r}-\frac{2(4+3 r)}{3+2 A}\right\},
$$

which exhibit behaviours that are rather close to those of curvatons with quadratic potentials. However, here the spectral index (2.45) vanishes at leading order, when $\dot{H}_{*}=0$. 


\section{References}

[1] A. A. Starobinsky, "A New Type of Isotropic Cosmological Models Without Singularity," Phys. Lett. B 91, 99 (1980).

[2] K. Sato, "First Order Phase Transition of a Vacuum and Expansion of the Universe," Mon. Not. Roy. Astron. Soc. 195, 467 (1981).

[3] A. H. Guth, "The Inflationary Universe: A Possible Solution to the Horizon and Flatness Problems," Phys. Rev. D 23, 347 (1981).

[4] S. Mukohyama, "Scale-invariant cosmological perturbations from Horava-Lifshitz gravity without inflation," JCAP 0906, 001 (2009) [arXiv:0904.2190 [hep-th]].

[5] V. A. Rubakov, "Harrison-Zeldovich spectrum from conformal invariance," JCAP 0909, 030 (2009) [arXiv:0906.3693 [hep-th]].

[6] P. Creminelli, A. Nicolis and E. Trincherini, "Galilean Genesis: An Alternative to inflation," JCAP 1011, 021 (2010) [arXiv:1007.0027 [hep-th]].

[7] A. D. Linde and V. F. Mukhanov, "Nongaussian isocurvature perturbations from inflation," Phys. Rev. D 56, 535 (1997) [arXiv:astro-ph/9610219].

[8] K. Enqvist and M. S. Sloth, "Adiabatic CMB perturbations in pre - big bang string cosmology," Nucl. Phys. B 626, 395 (2002) [arXiv:hep-ph/0109214].

[9] D. H. Lyth and D. Wands, "Generating the curvature perturbation without an inflaton," Phys. Lett. B 524, 5 (2002) [arXiv:hep-ph/0110002].

[10] T. Moroi and T. Takahashi, "Effects of cosmological moduli fields on cosmic microwave background," Phys. Lett. B 522, 215 (2001) [Erratum-ibid. B 539, 303 (2002)] [arXiv:hep-ph/0110096].

[11] K. Enqvist, S. Nurmi, JCAP 0510, 013 (2005). [astro-ph/0508573].

[12] K. Enqvist and T. Takahashi, "Signatures of Non-Gaussianity in the Curvaton Model," JCAP 0809, 012 (2008) [arXiv:0807.3069 [astro-ph]].

[13] K. Enqvist, S. Nurmi, G. Rigopoulos, O. Taanila and T. Takahashi, "The Subdominant Curvaton," JCAP 0911, 003 (2009) [arXiv:0906.3126 [astro-ph.CO]].

[14] M. Kawasaki, K. Nakayama, F. Takahashi, "Hilltop Non-Gaussianity," JCAP 0901, 026 (2009). [arXiv:0810.1585 [hep-ph]].

[15] M. Sasaki, J. Valiviita and D. Wands, "Non-Gaussianity of the primordial perturbation in the curvaton model," Phys. Rev. D 74, 103003 (2006) [arXiv:astro-ph/0607627]. 
[16] D. Larson et al., "Seven-Year Wilkinson Microwave Anisotropy Probe (WMAP) Observations: Power Spectra and WMAP-Derived Parameters," Astrophys. J. Suppl. 192, 16 (2011) [arXiv:1001.4635 [astro-ph.CO]].

[17] J. Dunkley et al., "The Atacama Cosmology Telescope: Cosmological Parameters from the 2008 Power Spectra," arXiv:1009.0866 [astro-ph.CO].

[18] R. Hlozek et al., "The Atacama Cosmology Telescope: a measurement of the primordial power spectrum," arXiv:1105.4887 [astro-ph.CO].

[19] D. H. Lyth, "What would we learn by detecting a gravitational wave signal in the cosmic microwave background anisotropy?," Phys. Rev. Lett. 78, 1861 (1997) [arXiv:hep$\mathrm{ph} / 9606387]$.

[20] K. Hamaguchi, M. Kawasaki, T. Moroi, F. Takahashi, "Curvatons in supersymmetric models," Phys. Rev. D69, 063504 (2004). [hep-ph/0308174].

[21] T. Kobayashi, S. Mukohyama, "Curvatons in Warped Throats," JCAP 0907, 032 (2009). [arXiv:0905.2835 [hep-th]].

[22] C. P. Burgess, M. Cicoli, M. Gomez-Reino, F. Quevedo, G. Tasinato and I. Zavala, "Non-standard primordial fluctuations and nongaussianity in string inflation," JHEP 1008, 045 (2010) [arXiv:1005.4840 [hep-th]].

[23] K. Dimopoulos, D. H. Lyth, A. Notari, A. Riotto, "The Curvaton as a pseudoNambuGoldstone boson," JHEP 0307, 053 (2003). [hep-ph/0304050].

[24] K. Dimopoulos, G. Lazarides, D. Lyth and R. Ruiz de Austri, "Curvaton dynamics," Phys. Rev. D 68, 123515 (2003) [arXiv:hep-ph/0308015].

[25] A. A. Starobinsky, "Multicomponent de Sitter (Inflationary) Stages and the Generation of Perturbations," JETP Lett. 42, 152 (1985) [Pisma Zh. Eksp. Teor. Fiz. 42, 124 (1985)].

[26] M. Sasaki and E. D. Stewart, "A General Analytic Formula For The Spectral Index Of The Density Perturbations Produced During Inflation," Prog. Theor. Phys. 95, 71 (1996) [arXiv:astro-ph/9507001].

[27] D. Wands, K. A. Malik, D. H. Lyth and A. R. Liddle, "A new approach to the evolution of cosmological perturbations on large scales," Phys. Rev. D 62, 043527 (2000) [arXiv:astroph/0003278].

[28] D. H. Lyth, K. A. Malik and M. Sasaki, "A general proof of the conservation of the curvature perturbation," JCAP 0505, 004 (2005) [arXiv:astro-ph/0411220].

[29] E. Komatsu and D. N. Spergel, "Acoustic signatures in the primary microwave background bispectrum," Phys. Rev. D 63, 063002 (2001) [arXiv:astro-ph/0005036]. 
[30] M. Kawasaki, K. Kohri, N. Sugiyama, "Cosmological constraints on late time entropy production," Phys. Rev. Lett. 82, 4168 (1999). [astro-ph/9811437].

[31] M. Kawasaki, K. Kohri, N. Sugiyama, "MeV scale reheating temperature and thermalization of neutrino background," Phys. Rev. D62, 023506 (2000). [astro-ph/0002127].

[32] S. Hannestad, "What is the lowest possible reheating temperature?," Phys. Rev. D 70, 043506 (2004).

[33] K. Ichikawa, M. Kawasaki and F. Takahashi, "The oscillation effects on thermalization of the neutrinos in the universe with low reheating temperature," Phys. Rev. D 72, 043522 (2005).

[34] E. W. Kolb, A. Notari and A. Riotto, "On the reheating stage after inflation," Phys. Rev. D 68, 123505 (2003) [arXiv:hep-ph/0307241].

[35] J. Yokoyama, "Can oscillating scalar fields decay into particles with a large thermal mass?," Phys. Lett. B 635, 66 (2006) [arXiv:hep-ph/0510091].

[36] M. Drewes, "On the Role of Quasiparticles and thermal Masses in Nonequilibrium Processes in a Plasma," arXiv:1012.5380 [hep-th].

[37] M. Fukugita, T. Yanagida, "Baryogenesis Without Grand Unification," Phys. Lett. B174, 45 (1986); For a review, see W. Buchmuller, R. D. Peccei, T. Yanagida, "Leptogenesis as the origin of matter," Ann. Rev. Nucl. Part. Sci. 55, 311-355 (2005). [hep$\mathrm{ph} / 0502169]$.

[38] T. Asaka, K. Hamaguchi, M. Kawasaki and T. Yanagida, "Leptogenesis in inflaton decay," Phys. Lett. B 464, 12 (1999) [arXiv:hep-ph/9906366]; "Leptogenesis in inflationary universe," Phys. Rev. D 61, 083512 (2000) [arXiv:hep-ph/9907559].

[39] I. Affleck and M. Dine, "A New Mechanism For Baryogenesis," Nucl. Phys. B 249, 361 (1985).

[40] M. Dine, L. Randall and S. D. Thomas, "Baryogenesis From Flat Directions Of The Supersymmetric Standard Model," Nucl. Phys. B 458, 291 (1996) [arXiv:hep-ph/9507453].

[41] J. M. Cline and S. Raby, "Gravitino induced baryogenesis: A Problem made a virtue," Phys. Rev. D 43, 1781 (1991).

[42] S. Weinberg, "Anthropic Bound on the Cosmological Constant," Phys. Rev. Lett. 59, 2607 (1987).

[43] F. C. Adams, J. R. Bond, K. Freese, J. A. Frieman and A. V. Olinto, "Natural inflation: Particle physics models, power law spectra for large scale structure, and constraints from COBE," Phys. Rev. D 47, 426 (1993) [arXiv:hep-ph/9207245]. 
[44] X. Wang, B. Feng, M. Li, X. L. Chen and X. Zhang, "Natural inflation, Planck scale physics and oscillating primordial spectrum," Int. J. Mod. Phys. D 14, 1347 (2005) [arXiv:astro-ph/0209242].

[45] B. Feng, M. z. Li, R. J. Zhang and X. m. Zhang, "An inflation model with large variations in spectral index," Phys. Rev. D 68, 103511 (2003) [arXiv:astro-ph/0302479].

[46] X. Chen, R. Easther and E. A. Lim, "Generation and Characterization of Large NonGaussianities in Single Field Inflation," JCAP 0804, 010 (2008) [arXiv:0801.3295 [astro$\mathrm{ph}]]$.

[47] C. Pahud, M. Kamionkowski and A. R. Liddle, "Oscillations in the inflaton potential?," Phys. Rev. D 79, 083503 (2009) [arXiv:0807.0322 [astro-ph]].

[48] J. Hamann, S. Hannestad, M. S. Sloth and Y. Y. Y. Wong, "Observing trans-Planckian ripples in the primordial power spectrum with future large scale structure probes," JCAP 0809, 015 (2008) [arXiv:0807.4528 [astro-ph]].

[49] R. Flauger, L. McAllister, E. Pajer, A. Westphal and G. Xu, "Oscillations in the CMB from Axion Monodromy Inflation," JCAP 1006, 009 (2010) [arXiv:0907.2916 [hep-th]].

[50] S. Hannestad, T. Haugbolle, P. R. Jarnhus and M. S. Sloth, "Non-Gaussianity from Axion Monodromy Inflation," JCAP 1006, 001 (2010) [arXiv:0912.3527 [hep-ph]].

[51] R. Flauger and E. Pajer, "Resonant Non-Gaussianity," JCAP 1101, 017 (2011) [arXiv:1002.0833 [hep-th]].

[52] T. Kobayashi and F. Takahashi, "Running Spectral Index from Inflation with Modulations," JCAP 1101, 026 (2011) [arXiv:1011.3988 [astro-ph.CO]].

[53] J. Garcia-Bellido, D. Wands, "The Spectrum of curvature perturbations from hybrid inflation," Phys. Rev. D54, 7181-7185 (1996). [astro-ph/9606047].

[54] W. H. Kinney, "A Hamilton-Jacobi approach to nonslow roll inflation," Phys. Rev. D56, 2002-2009 (1997). [hep-ph/9702427].

[55] A. D. Linde, "Fast roll inflation," JHEP 0111, 052 (2001). [hep-th/0110195].

[56] W. H. Kinney, "Horizon crossing and inflation with large eta," Phys. Rev. D72, 023515 (2005). [gr-qc/0503017].

[57] K. Tzirakis, W. H. Kinney, "Inflation over the hill," Phys. Rev. D75, 123510 (2007). [astro-ph/0701432].

[58] L. Kofman, S. Mukohyama, "Rapid roll Inflation with Conformal Coupling," Phys. Rev. D77, 043519 (2008). [arXiv:0709.1952 [hep-th]]. 
[59] T. Kobayashi, S. Mukohyama, B. A. Powell, "Cosmological Constraints on Rapid Roll Inflation," JCAP 0909, 023 (2009). [arXiv:0905.1752 [astro-ph.CO]]. 